JOURNAL OF THE

AMERICAN MATHEMATICAL SOCIETY

Volume 9, Number 3, July 1996

\title{
THE MORDELL-LANG CONJECTURE FOR FUNCTION FIELDS
}

\author{
EHUD HRUSHOVSKI
}

\section{INTRODUCTION}

In [La65], Lang formulated a hypothesis including as special cases the Mordell conjecture concerning rational points on curves, and the Manin-Mumford conjecture on torsion points of Abelian varieties. Sometimes generalized to semi-Abelian varieties, and to positive characteristic, this has been called the Mordell-Lang conjecture; see [AV91] and [La91]. It is essentially a finiteness statement on the intersection of a subvariety of a semi-Abelian variety with a subgroup of finite rank. We prove here the function-field version of the conjecture, in any characteristic.

In characteristic 0, the Mordell-Lang conjecture was proved in a sequence of papers by Raynaud, Faltings and Vojta, at least in the case of Abelian varieties or finitely generated groups. The full result was proved over number fields, and these cases were inferred for function fields using a specialization argument; see [La91] for a description. For Abelian varieties in characteristic 0, a quite different argument was found by Buium; this inspired our approach. In positive characteristic, many cases were proved in [AV91]. This paper presents a uniform proof incorporating all cases, using model theoretic ideals. We describe the strategy following the statement of the result (equivalent to the statement in [AV91]).

In this statement, and in the entire paper, we use the language of varieties rather than schemes. We refer to the absolute Zariski topology unless otherwise stated, and generally use terms in their geometric sense (over an algebraically closed field).

Let $K / k$ be a field extension, $k$ algebraically closed. Call a group $\Gamma p^{\prime}$-finitely generated if $Q_{p} \otimes \Gamma$ is finitely generated as a $Q_{p}$-module, where $Q_{p}=\mathbf{Q}$ if $p=0$, and $Q_{p}=\{m / n \in \mathbf{Q}: n$ prime to $p\}$ if $p>0$. This condition is of course valid for finitely generated Abelian groups, and for prime-to- $p$-torsion groups. Recall that a semi-Abelian variety is an extension of an Abelian variety by an algebraic torus.

Theorem 1.1. Let $S$ be a semi-Abelian variety defined over $K$, and $X$ a subvariety of $S$. Let $\Gamma$ be a $p^{\prime}$-finitely generated subgroup of $S$. Suppose $X \cap \Gamma$ is Zariski dense in $X$. Then there exists a semi-Abelian variety $S_{0}$ defined over $k$, a subvariety $X_{0}$ of $S_{0}$ defined over $k$, and a rational homomorphism $h$ from a group subvariety of $S$ into $S_{0}$, such that $X$ is a translate of $h^{-1}\left(X_{0}\right)$.

Call a subvariety of $S$ satisfying the conclusion special. The theorem then states that for any subvariety $Z$ of $S, Z \cap \Gamma$ is contained in a finite union of special

Received by the editors September 1, 1993 and, in revised form, November 1, 1994.

1991 Mathematics Subject Classification. Primary 03C45, 11G10; Secondary 03C60, 14G05, $12 \mathrm{H} 05$.

The author was supported by the National Science Foundation.

(C)1996 American Mathematical Society 
subvarieties of $Z$. (To see this, apply the theorem to the irreducible components $X$ of the Zariski closure of $Z \cap \Gamma$.) If $S$ is an Abelian variety with $K / k$ trace 0 , the special subvarieties are just the cosets of Abelian subvarieties of $S$. In this case the theorem reads as follows.

Corollary 1.2. Let $k$ be an algebraically closed field, $\underline{A}$ an Abelian variety with no nonzero homomorphic images defined over $k, \underline{X}$ a subvariety of $\underline{A}$, and $\Gamma$ a $p^{\prime}$-finitely generated subgroup of $A$. Then $\underline{X}(K) \cap \Gamma=\underline{Y}(K) \cap \Gamma$, where $\underline{Y}$ is a (reducible) subvariety of $\underline{X}$, equal to a finite union of translates of Abelian subvarieties of $\underline{A}$.

See also $\S 6$ for some quantitative statements, one in the presence of a discrete valuation of $K / k$ and hence a distance function, another giving an exponential bound on the number of translates in 1.2 in terms of the rank of $\Gamma$ (the other data being fixed), in fixed positive characteristic.

We remark here that the characteristic zero geometric Mordell-Lang conjecture can be deduced by specialization arguments from the positive characteristic case. This was suggested in [La91], following the description of Voloch's theorem; Lang says one would need to know something on existence of ordinary specializations, but that's because of the ordinariness hypothesis in Voloch's result, no longer present. (Some other issues arising from the higher dimension and non-finite-generation need to be addressed, but this can be done using methods of Neron and Raynaud.) However we will not take this route, and will give a direct proof in characteristic zero. The exponential growth with $\operatorname{rk}(\Gamma)$ alluded to above does not appear to ascend to characteristic zero by this method.

Our opening move is taken from Buium ([Bu92]), who proved certain cases of the theorem, including Corollary 1.2 in characteristic 0, using differential algebra. A differential field should be regarded as an infinite-dimensional object, and in particular an Abelian variety can no longer be viewed as finite-dimensional in an absolute sense. However, using a homomorphism introduced by Manin ([Ma58], [Ma63]), Buium points out that $\Gamma$ is contained in a certain finite-dimensional group. At this point he proceeds to use some of his theory of finite-dimensional Kolchin closed groups (but also some analysis). Our proof uses instead the model theory of abstract finite-dimensional groups ("groups of finite Morley rank").

An algebraic structure (e.g. a group with a distinguished subset) is said to have finite Morley dimension if one can assign an integer dimension to the "definable subsets" so that certain natural conditions are satisfied. Such structures have been analyzed, in the abstract, by model theorists, motivated initially by categoricity questions. Examples are provided by algebraic varieties over an algebraically closed field, with the usual dimension theory. However we apply the theory to the kernel of the Manin homomorphism and certain related groups, that do not a priori carry the structure of an algebraic variety. Following some reductions, we apply a general dichotomy theorem of B. Zilber and the author. This theorem implies that an enriched group satisfying the appropriate dimension-theoretic axioms is either a module over a certain local ring, with no additional structure, or it carries the structure of an algebraic group over an algebraically closed field. This dichotomy leads to the two kinds of subvarieties mentioned implicitly in the theorem: group subvarieties, and ones arising from varieties defined over the (algebraically closed) constant field. 
In positive characteristic, we do not explicitly use differential algebra. We work instead with fields $K$ of some fixed finite dimension over $K^{p}$, endowed with a distinguished basis for $K / K^{p}$. The role of the kernel of the Manin homomorphism is played by the group $p^{\infty} A(F)$ of infinitely $p$-divisible points; $F$ is a large field that will be described below. It can be shown that for each $n$, there is a map from $A(F)$ into a vector group over the truncated Witt vector ring $W_{n}(F)$ whose kernel is $p^{n} A(F)$ (up to finite index). We note that Manin asks in [Ma58] for a positive characteristic analog of the Manin homomorphism. We are not certain if we have found the "correct" analog. For us only the kernel $p^{\infty} A(F)$ plays a role; we show in $\S 2$ that it has finite Morley dimension, and enjoys a dimension theory as in the characteristic 0 case. Beyond this point, the proof is uniform in the different characteristics. (Indeed it appears that the divergence in the proofs in the different characteristics is due merely to an accident of the historical development of model theory. Distinguishing a basis for $K / K^{p}$ has the effect of fixing also a stack of Hasse derivations. One expects that quantifier elimination and elimination of imaginaries hold already in the differential language, without the distinguished basis, and in this language the proof should become entirely uniform with respect to the characteristic.)

We refer the reader to the short expository paper [NeP89] for basic modeltheoretic notions, and to [Sa72] for proofs. The paper [HZ] also contains a short section summarizing some of the relevant definitions.

We proceed to describe sequentially the organization of the paper. We work throughout in a universal domain $F$. If the characteristic is $0, F$ is a field with a distinguished derivation. If it is $p, F$ is a field with a distinguished $p$-basis (i.e. a distinguished basis of $F$ over $F^{p}$ ); the basis is assumed finite, with $p^{\nu}$ elements. In either case we make the following assumption:

(*) Let $F_{0}$ be a countable differential field (respectively, a countable field of characteristic $p$ with distinguished $p$-basis of size $p^{\nu}$ ). Then $F_{0}$ embeds into $F$ (preserving the derivation, or the $p$-basis). Moreover, any such embedding is unique, up to an automorphism of $F$.

Such structures are called $\lambda$-saturated $(\lambda>\omega)$. They can be shown to exist by standard model theoretic methods; see [Sa72] $(\S 16, \S 40)$ and [Del88], or [RR75]. (They also enjoy strong uniqueness properties, whose discussion however would be irrelevant here.) One immediate example of their usefulness for us is furnished by the following observation. Whereas in general the group of infinitely-p-divisible points of an Abelian variety over a separably closed field may be trivial, in the saturated model the group is large, and reflects to a certain extent the properties of the ambient Abelian variety.

In $\S 2$ we discuss the appropriate dimension theory, and describe a context in which it applies, over separably closed fields. In particular we deduce that the group of infinitely- $p$-divisible points mentioned above falls into this framework (Lemma 2.15).

In $\S 3$ we state the main theorem in the language of differentially closed fields (following Buium's lead), or in the language of separably closed fields (in characteristic $p>0)$. We show that it implies Theorem 1.1 as stated.

In $\S 4$ we develop the required theory of Abelian groups of finite Morley dimension. The results of this section apply of course to commutative algebraic groups, but the main issues dealt with here do not exist in that context. Better examples may be found in the domain of complex tori; these can have some subquotients 
that are Abelian varieties and others that are not; the interaction between them, in the general context, forms the subject of study of the section.

In $\S 5$ we prove the main theorem. It is here that we use the powerful results from [HZ]. These results apply at or near dimension one, and it is the theory of $\S 4$ that permits the reduction to that level.

In $\S 6$ we give a quantitative variant of the result, conjectured by Voloch, in the presence of a valuation and a corresponding local proximity function. In the situation of Corollary 1.2, we show that the distance from a point of $\Gamma$ to $X$ can be bounded in terms of its distance to $Y$.

I would like to thank Zoe Chatzidakis, Elisabeth Bouscaren, Anand Pillay, and Carol Wood for their reading of this paper.

\section{Thin types And Zariski geOMetries}

The goal of this section is to introduce the various notions of finite dimension, and to show that the types inside the kernel group admit a dimension theory of the required type. The facts in characteristic 0 are largely classical model theory, see e.g. [Sa72]. The fact that minimal types are Zariski in the sense below (after removing a finite number of singularities) is shown in [HS]. Following the initial definitions, we will therefore concentrate on characteristic $p>0$.

We work in a universal domain $U$. acl denotes algebraic closure in the model theoretic sense; so for countable $X, \operatorname{acl}(X)$ is the set of elements of $U$ whose orbit under $\operatorname{Aut}(U / X)$ is finite. $\operatorname{dcl}(X)$ is the set of elements definable over $X$, or the set of elements of $U$ fixed by $\operatorname{Aut}(U / X)$. If $U$ is an algebraically closed field and $\langle X\rangle$ is the subfield generated by a subset $X$ of $U$, then $\operatorname{acl}(X)$ is the algebraic closure of $\langle X\rangle$ in $U$, and $\operatorname{dcl}(X)$ is the perfect closure of $\langle X\rangle$.

We need to lightly modify standard model theoretic usage in order to make the language of Morley dimension apply in our context.

Definition 2.1. Let $P$ be the solution set to a set of formulas of size $\leq \lambda$, in a $\lambda^{+}$-saturated model. A definable subset of $P$ is a set of the form $D \cap P$, where $D$ is a definable set (perhaps with parameters outside $P$ ). $P$ is minimal if every definable subset of $P$ is finite or cofinite. Let $B$ be any set over which $P$ is defined; the following notions are relative to $B$. If $A$ is a small set of elements of $P$, let the rank $\operatorname{rk}_{P}(A)$ be the size of any maximal algebraically independent subset of $A$. In general, let $\operatorname{rk}_{P}(A)=\operatorname{rk}_{P}(\operatorname{acl}(A) \cap P)$. If $q=\operatorname{tp}\left(a_{1}, \ldots, a_{m}\right)$, let $\operatorname{rk}^{P}(q)=\operatorname{rk}\left(\left\{a_{1}, \ldots, a_{m}\right\}\right)$. If $D$ is a definable or $\infty$-definable subset of $P^{n}$, define $\operatorname{rk}^{P}(D)=\max \{\operatorname{rk}(q): q$ a type in $D$ \}; an element of maximal rank of $D$ is called generic. We omit $P$ if its identity is clear.

$P$ is called pluriminimal if it is a finite union of minimal types. It is called semiminimal if there exists a minimal type $Q$ and a finite set $F$ such that $P \subseteq \operatorname{acl}(F, Q)$. Similarly define semi-pluriminimal.

Morley dimension is defined recursively as follows. $P$ is said to have dimension -1 iff it is empty. $P$ has dimension $k$ if for some integer $m, P$ cannot be split into $m+1$ definable subsets none of which have dimension $\leq(k-1)$. The smallest such $m$ is called the Morley degree, or multiplicity, of $P$. (If $U$ is an algebraically closed field, this agrees with Zariski dimension.)

Lemma 2.2 ("internal Morley dimension"). Let $P$ be minimal, and let $D$ be a definable subset of $P^{n}$ of rank $k$. There exists an integer $m=\operatorname{Mult}(D)$ such that $D$ cannot be split into $m+1$ pairwise disjoint definable subsets of rank $k$. 
Proof. It suffices to show that there are only finitely many types $q$ in $D$ with $\operatorname{rk}(q)=k$. Let $p^{[k]}$ be the type of a generic $k$-tuple from $P$. For each subset $s$ of $\{1, \ldots, n\}$ of size $k$, consider the partial type $q_{s}\left(x_{1}, \ldots, x_{n}\right)$ asserting that $p^{[k]}$ holds of $\left(x_{i}: i \in s\right),\left(x_{1}, \ldots, x_{n}\right) \in D$, and each $x_{i} \in P$. Let $a_{i}(i \in s)$ realize $p^{[k]}$. Then there are only finitely many choices of $a_{i}(i \notin s)$ such that $\left(a_{i}: i=1, \ldots, n\right)$ realizes $q_{s}$. For otherwise there would be such a choice $\left(a_{1}, \ldots, a_{n}\right)$ with some $a_{j}$ $(j \notin s)$ nonalgebraic over $\left(a_{i}: i \in s\right)$, so $\operatorname{rk}\left(a_{1}, \ldots, a_{n}\right) \geq k+1$, contradicting $\operatorname{rk}(D)=k$. Thus each $q_{s}$ has only finitely many complete extensions. Since every rank- $k$ extension of $D$ extends some $q_{s}$, there are only finitely many of these.

The lemma shows that for definable subsets of $P^{n}$, dimension equals rank. (If $P$ is minimal.)

Definition 2.3. Let $T$ be a theory with quantifier elimination and $P$ a minimal type. $P$ is called Zariski if (i)-(iii) hold. (iii) is referred to as the "dimension theorem".

Call a subset of $P^{n}$ closed if it is defined by a positive, quantifier-free formula. Call it irreducible if it is not the union of two proper closed subsets.

(i) Every closed set in $P^{n}$ is the union of finitely many closed, irreducible sets.

(ii) If $X$ is a proper subset of $Y$, both closed subsets of $P^{n}$, and $Y$ is irreducible, then $\operatorname{rk}(X)<\operatorname{rk}(Y)$.

(iii) If $X$ is a closed, irreducible subset of $P^{n}, \operatorname{rk}(X)=m$, and $Y$ is a diagonal $x_{i}=x_{j}$, then $X \cap Y$ is the union of closed irreducible sets of dimension at least $m-1$.

Remark 2.4. Under these conditions, the collection of closed sets of $P^{n}$ defines a Noetherian topology, and the dimension of a closed set with respect to this topology equals its rank. For every definable set $Y$ there exists a proper closed subset $F$ of $\operatorname{cl}(Y)$ such that $Y-F=\operatorname{cl}(Y)-F$.

Proof. The first statement is clear: given an infinite descending chain of closed sets, the first can be assumed irreducible, hence the second has smaller rank, beginning an infinite descent of ranks. If $X$ is irreducible, of rank $k$, one shows $\operatorname{dim}(X) \geq k$ by induction on $k: X$ has a subset of rank $k-1$; this subset can be chosen closed, irreducible, hence by induction has dimension $k-1$, so $X$ has dimension $\geq k$. The other inequality is immediate from (ii). For the final statement, note that $Y$ is a finite union of sets $Y_{i}=H_{i}-F_{i}$, with $H_{i}$ closed irreducible and $F_{i}$ a proper closed subset. We may assume $Y$ is not the union of fewer of these. Let $H=\bigcup_{i} H_{i}$, $F=\bigcup_{i} F_{i}$. Then $\operatorname{cl}(Y)=H$; and some $H_{i}$ is not contained in any other $H_{j}$, hence not in $F_{i}$, and being irreducible, not in $F$. Thus $F$ is a proper subset of $H$, and $H-F \subseteq Y$.

Lemma 2.5. Let $T$ be a stable theory with a minimal type $P$. Assume $P$ is Zariski and not locally modular. Then $T$ interprets a field $F$, with definable subfields $F_{\alpha}$, such that $\bigcap_{\alpha} F_{\alpha}$ is minimal, and nonorthogonal to $P$.

Proof. This is proved in [HZ] when $P$ is strongly minimal (i.e. it is the solution set of a single formula), but the proof goes through, and gives a type-definable field $F^{*}$, minimal and nonorthogonal to $P$. By [Hr90], there exists a definable field $F$ and definable subfields $F_{\alpha}$, such that $\bigcap_{\alpha} F_{\alpha}=F^{*}$.

We require here only the results of $\S 6$ of [HZ]. This section is written largely axiomatically, the axioms having been proved in $\S 4$ and $\S 5$. At the end of this 
section (2.20), we will indicate how one can shortcut this and directly prove the axioms in the present contex.

2.6 Conventions on separably closed fields. Let $T(p, \nu)$ be the theory of separably closed fields $F$ of char $p>0$, of finite dimension $p^{\nu}$ over $F^{p}$. ( $\nu$ is the Ersov invariant.) We endow $F$ with a basis $e_{1}, \ldots, e_{p^{\nu}}$ of $F$ over $F^{p}$. The language is the language of rings with $p^{\nu}$ distinguished constants, for the $e_{i}$, as well as function symbols for certain definable functions $\lambda_{n}$, described in 2.7(a) below. We work in a universal domain $U=F$ for this theory (a countably saturated model). The notions of substructure and algebraic closure will be relative to $F$, in this language.

Usually we will denote algebraic varieties defined over $F$ by an underlined capital letter, such as $\underline{V}$, and the group of $F$-points of $\underline{V}$ by $V$. We often implicitly assume that $\underline{V}$ is given with an affine chart, and so we discuss coordinates of elements of $\underline{V}$; in particular the set of $F$-points makes sense. We can also apply the Frobenius to $\underline{V}$. The variety obtained in this way will be denoted $\operatorname{Fr} \bar{V}$. If $V$ is a definable group, the set of $p$-th powers of elements of $V$ will be denoted $V^{p}$. [ $[V]^{p}$ denotes the set of $p$-tuples from $V$. When there is no danger of confusion with the previous two meanings, we revert to the notation $V^{p} . T(p, \nu)$ admits quantifier elimination and elimination of imaginaries (see [Del88]).

Fact 2.7 ([Del88]). In $T(p, \nu)$ there exist (basic) definable functions $\lambda_{n}: F \rightarrow F^{p^{\nu n}}$ with the following properties:

(a) $\lambda_{n}$ is the inverse of the bijective morphism $r\left(x_{1}, \ldots, x_{p^{\nu n}}\right)=\sum x_{i}^{p^{n}} e_{n, i}$ for some designated basis $\left\{e_{n, i}\right\}$ of $F$ over $F^{p^{n}}$. In particular, $\lambda_{n}=r_{n} \circ \lambda_{n+1}$ for a certain polynomial function $r_{n}:[F]^{p^{\nu(n+1)}} \rightarrow[F]^{p^{\nu n}}$.

(b) Any atomically definable subset of $[F]^{k}$ is, for some $n$, the pullback by $\lambda_{n}^{-1}$ of some (possibly reducible) subvariety $U$ of $\left[F^{p^{\nu n}}\right]^{k}$. Let $\operatorname{dcl}_{f}(X)$ denote the field generated by $X \cup\left\{e_{1}, \ldots, e_{p^{\nu}}\right\}$, and $\operatorname{acl}_{f}(X)$ the relative field-theoretic algebraic closure of $\operatorname{dcl}_{f}(X)$ in the field $F$.

(c) If $a \in \operatorname{acl}\left(b_{1}, \ldots, b_{d}\right)$, then $a \in \operatorname{acl}_{f}\left(\left\{\lambda_{n} b_{i}: i \leq d, n<\infty\right\}\right)$.

(d) If $a \in \operatorname{dcl}\left(b_{1}, \ldots, b_{d}\right)$, then $a \in \operatorname{dcl}_{f}\left(\left\{\lambda_{n} b_{i}: i \leq d, n<\infty\right\}\right)$.

Proof. (c) $\operatorname{acl}_{f}\left(\left\{\lambda_{n} b_{i}: i \leq d, n<\infty\right\}\right)$ is an elementary substructure of $F$, so it is (model-theoretically) algebraically closed.

(d) Suppose $a \in \operatorname{dcl}\left(b_{1}, \ldots, b_{d}\right)$. Let $k=\operatorname{dcl}_{f}\left(\left\{\lambda_{n} b_{i}: i \leq d, n<\infty\right\}\right)$. Note that $k$ is a perfect field. The separable closure $k_{s}$ of $k$ is an elementary submodel of $F$, so $a \in k_{s}$, and every automorphism of $k_{s}$ fixing $k$ also fixes $a$. Hence $a \in k$.

Definition 2.8. $\operatorname{tp}(c / B)$ is $(k$ - $)$ thin if $\operatorname{dcl}(c, B)$ is a field extension of $\operatorname{dcl}(B)$ of finite transcendence degree (at most $k$ ).

Lemma 2.9. Let $K$ be a countable subfield of $F, K=\operatorname{dcl}(K) \cdot \operatorname{tp}(a / K)$ is $k$-thin iff the following condition holds:

(*) for all powers $q$ of $p=\operatorname{char}(F)$ there exists a subfield $L$ of $F$ containing $K$ and of transcendence degree $\leq k$ over $K$, such that $a \in K L^{q}$.

Proof. If $\operatorname{tp}(a / K)$ is $k$-thin, given $q=p^{n}$, let $L=K\left(\lambda_{n}(a)\right)$. Then since each basis element of $F / F^{q}$ lies in $K, a \in K L^{q}$. For the converse, we require a claim.

Claim. If $a \in K L^{q}$ and $q=p^{n+m}$, then each coordinate of $\lambda_{n}(a)$ is in $K L^{p^{m}}$.

Proof. We have $a=f(b) / g(b)$, where $f, g \in K[X]$, a polynomial ring, and $b$ is from $L^{q}$. Let $c \in L^{p^{m}}$ be such that $c^{p^{n}}=b$. We can write $a=f(b) g(b)^{p^{n-1}} / g(b)^{p^{n}}$, 
so we may assume $g \in K[X]^{p^{n}}$. Let $\left\{e_{i}\right\}$ be the chosen basis for $F / F^{p^{n}}$, and write $f=\sum e_{i} f_{i}^{\left[p^{n}\right]}$, where $f_{i} \in F[X]$ and $f_{i}^{\left[p^{n}\right]}$ is the result of taking the $p^{n}$-th power of each coefficient. Since $K=\operatorname{dcl}(K), f_{i} \in K[X]$ for each $i$. Clearly

$$
\begin{aligned}
\lambda_{n}(a) & =\lambda_{n}(f(b)) / g(b)=\lambda_{n}\left(\sum e_{i} f_{i}^{\left[p^{n}\right]}\left(c^{p^{n}}\right)\right) / g(b) \\
& =\lambda_{n}\left(\sum e_{i} f_{i}(c)^{p^{n}}\right) / g(b)=\left(f_{1}(c) / g(b), \ldots, f_{p^{\nu n}}(c) / g(b)\right) .
\end{aligned}
$$

Evidently all the coordinates are in $K L^{p^{m}}$.

Now if $(*)$ holds, let $L_{n}$ witness $(*)$ for $q=p^{n}$. Then $\operatorname{tr} \operatorname{deg}(\operatorname{dcl}(a) / K)=$ $\sup \operatorname{tr} \operatorname{deg} K\left(\lambda_{n}(a)\right) / K \leq \operatorname{tr} \operatorname{deg} L_{n} / K \leq k$.

Lemma 2.10. (i) If $\operatorname{tp}(a / K)$ is $k$-thin, $K \subseteq K^{\prime}$, then $\operatorname{tp}\left(a / K^{\prime}\right)$ is $k$-thin.

(ii) If $\operatorname{tp}\left(a_{i} / K\right)$ is $k_{i}$-thin, $a \in \operatorname{acl}_{f}\left(a_{1}, \ldots, a_{m}\right), k=\sum k_{i}$, then $\operatorname{tp}(a / K)$ is $k$-thin.

(iii) If $\operatorname{tp}(a / K)$ is $k$-thin, then so is $\operatorname{tp}\left(\lambda_{n} a / K\right)$.

(iv) If $\operatorname{tp}\left(a_{i} / K\right)$ is $k_{i}$-thin, $a \in \operatorname{acl}\left(a_{1}, \ldots, a_{m}\right), k=\sum k_{i}$, then $\operatorname{tp}(a / K)$ is $k$-thin.

Proof. (i), (ii), (iii) are clear. (iv) follows from (ii), (iii), and (c) of Fact 2.7.

Recall the definition of $U(a / B)$ ([Las $])$. We say that $U(a / B)=0$ if $a \in \operatorname{acl}(B)$; $U(a / B) \leq n+1$ if for all $B^{\prime}$ containing $B$ such that a forks with $B^{\prime}$ over $B$, $U\left(a / B^{\prime}\right) \leq n ; U(a / B)=n$ if it is $\leq n$ but not $\leq(n-1)$. In the present context, " $a$ forks with $B^{\prime}$ over $B$ " means that $\operatorname{dcl}(a, B)$ is not free from $\operatorname{dcl}\left(B^{\prime}\right)$ over $\operatorname{dcl}(B)$; see [Del88]. Note that $U(a / B)=1$ iff $\operatorname{tp}(a / \operatorname{acl}(B))$ is minimal.

Lemma 2.11. If $\operatorname{tp}(a / B)$ is $k$-thin, then $U(a / B) \leq k$.

Proof. By induction on $k$, this is immediate from the above definitions.

Proposition 2.12. A thin minimal type in $T(p, \nu)$ is Zariski.

Proof. The assumption here is that the type is complete over some base substructure $K$.

The first claim takes place over an algebraically closed field.

Claim 2.12.1. Let $U, V$ be irreducible smooth varieties of the same dimension, and $f: U \rightarrow V$ a finite rational map (defined everywhere on $U$ ). Let $C$ be a closed irreducible subset of $V^{n}$. Then all components of $f^{-1} C$ have the same dimension (equal to $\operatorname{dim}(C)$ ).

Proof. The induced map $f: U^{n} \rightarrow V^{n}$ satisfies the same assumptions as $f$, so we may assume $n=1$. Note that the graph $F$ of $f$ is a closed irreducible subset of $U \times V$ (because we have a surjective rational map from $U$ to $F$ ). $f^{-1} C$ is isomorphic to $F \cap(U \times C)$. Let $X$ be a component of $F \cap(U \times C)$. By the dimension theorem on $U \times V$,

$$
\begin{aligned}
\operatorname{dim} X & \geq \operatorname{dim} F+\operatorname{dim}(U \times C)-\operatorname{dim}(U \times V) \\
& =\operatorname{dim}(U)+\operatorname{dim}(U)+\operatorname{dim}(C)-2 \operatorname{dim}(U)=\operatorname{dim}(C) .
\end{aligned}
$$

On the other hand the projection is a finite map from $X$ to $C$, so $\operatorname{dim} X \leq \operatorname{dim} C$. 
We now work in a universal domain for $T(p, \nu)$. Using elimination of imaginaries and the existence of a definable pairing function, it suffices to consider 1-types. Let $P$ be a 1-type over $K, K$ an algebraically closed, countable substructure of the universal domain.

Let $P_{n}$ be the Zariski closure of $\lambda_{n} P$, a subvariety of $[F]^{p^{\nu n}} \cdot r_{n}$ maps $P_{n+1}$ to $P_{n}$. Since $P$ is thin, for large enough $n$, say $n \geq N \#, r_{n}$ is finite-to-one above some Zariski open subset $P_{n}^{*}$ of $P_{n}$. We may choose $P_{n}^{*}$ smooth; and by defining $P_{n}^{*}$ inductively, we can arrange that $r_{n}$ carries $P_{n+1}^{*}$ into $P_{n}^{*}$. Let $e=\operatorname{dim} P_{n}$ for large $n$. If $U \subseteq P_{n}^{k}$, write $\lambda_{n}^{*} U$ for $\left\{x \in P^{k}: \lambda_{n} x \in U\right\}$, where $\lambda_{n}\left(x_{1}, \ldots, x_{k}\right)=$ $\left(\lambda_{n} x_{1}, \ldots, \lambda_{n} x_{k}\right)$.

Let $X$ be a closed subset of $P^{k}$. We will show that (i) of the definition of Zariski geometry holds by induction on $\operatorname{rk}(X)$. Let $X_{n}$ be the Zariski closure in $\left[P_{n}\right]^{k}$ of $\lambda_{n} X$. Then for all large enough $n, X=\lambda_{n}^{*} X_{n}$.

Claim 2.12.2. $\operatorname{dim}\left(X_{n}\right)=e \cdot \operatorname{rk}(X)$ for large $n$. (More specifically, for any $n \geq N \#$ such that $X=\lambda_{n}^{*} X_{n}$.)

Proof. Let $\left(a_{1}, \ldots, a_{k}\right)$ be a point of $X$, of $\operatorname{rank} d=\operatorname{rk}(X)$. Say $a_{1}, \ldots, a_{d}$ are algebraically independent, so $a \in \operatorname{acl}\left(a_{1}, \ldots, a_{d}\right)$. By (c), for all $k$ and $j, \lambda_{k} a_{j} \in$ $\operatorname{acl}_{f}\left(\left\{\lambda_{n} a_{i}: i \leq d, n<\infty\right\}\right)$. But since $P$ is thin, for some $N \#, \operatorname{acl}_{f}\left(\left\{\lambda_{n} a_{i}\right.\right.$ : $n<\infty\})=\operatorname{acl}_{f}\left(\lambda_{N \#} a_{i}\right)$. Thus for $n \geq N \#, \lambda_{n} a_{i} \in \operatorname{acl}_{f}\left(\lambda_{n} a_{1}, \ldots, \lambda_{n} a_{d}\right)$. Since this holds for any $\left(a_{1}, \ldots, a_{k}\right) \in X$, by compactness it holds in one of a finite number of ways, and so persists to the Zariski closure: $y_{i} \in \operatorname{acl}_{f}\left(y_{1}, \ldots, y_{d}\right)$ for any $\left(y_{1}, \ldots, y_{k}\right)$ in the Zariski closure $X_{n}$ of $\lambda_{n} X$, after some permutation of indices. Thus $\operatorname{dim} X_{n} \leq d \cdot \operatorname{dim}\left(P_{n}\right)$. On the other hand for $i \leq d$, the algebraic locus of $\lambda_{n} a_{i}$ over $\left\{\lambda_{n} a_{1}, \ldots, \lambda_{n} a_{i-1}\right\}$ contains $\lambda_{n} b$ for all but finitely many $b \in P$; hence it equals $P_{n}$. Thus

$$
\operatorname{dim} X_{n}=d \cdot \operatorname{dim}\left(P_{n}\right)=d e=e \cdot \operatorname{rk}(X) .
$$

We consider only $n \geq N \#$. Write $X_{n}=X_{n}(1) \cup \cdots \cup X_{n}\left(m_{n}\right) \cup Y_{n}$, where $X_{n}(i)$ are the distinct components of $X_{n}$ of dimension de. Let $X(n ; i)=\lambda_{n}^{*} X_{n}(i)$. Then $X_{n}(i)$ is the Zariski closure of $\lambda_{n} X(n ; i)$. By 2.12 .2 applied to $X(n ; i)$, we have $\operatorname{rk} X(n ; i)=\operatorname{dim} X_{n}(i) / e=\operatorname{rk}(X)$. Similarly the intersection of $X(n ; i)$ with $X\left(n ; i^{\prime}\right)\left(i \neq i^{\prime}\right)$ has smaller rank. By Lemma 2.2 , the number $m_{n}$ is bounded independently of $n$. By Claim $1, r_{n}^{-1} X_{n}(i)$ is a union of some of the components $X_{n+1}(j)$. So $m_{n}$ is nondecreasing with $n$; so for $n$ above some $N^{*}(\geq N \#)$ it reaches a constant maximum $m^{*}$. It follows that for $n \geq N^{*}, r_{n}^{-1} X_{n}(i)$ equals some $X_{n+1}(j)$; we recursively renumber so that $r_{n}^{-1} X_{n}(i)=X_{n+1}(i)$. So $X\left(N^{*} ; i\right)=$ $X\left(N^{*}+1 ; i\right)=\cdots \stackrel{\text { def }}{=} X(i)$. Let $Y=\lambda_{N^{*}}^{*} Y_{N^{*}}$. Then $X=X(1) \cup \cdots \cup X\left(m^{*}\right) \cup Y$, $Y$ is closed of rank smaller than $\operatorname{rk}(X)$, and $X(i)$ is closed of rank equal to $\operatorname{rk}(X)$. It remains only to show that $X(i)$ is irreducible. Suppose $X(i)=U \cup V$, with $U, V$ closed. Pick $n \geq N^{*}$ such that $U=\lambda_{n}^{*} U_{n}, V=\lambda_{n}^{*} V_{n}$, with $U_{n}, V_{n}$ the Zariski closures in $P_{n}^{* k}$ of $\lambda_{n} U, \lambda_{n} V$ respectively. Then $X_{n}(i)=U_{n} \cup V_{n}$. Since $X_{n}(i)$ is irreducible, it equals one of them; say $X_{n}(i)=U_{n}$. Then in $[P]^{k}$,

$$
X_{i}=\lambda_{n}^{*} X_{n}(i)=\lambda_{n}^{*} U_{n}=U
$$

We have shown that any closed set $X$ is a finite union of irreducible components. Further the proof showed that if $X$ is irreducible, then $X=\lambda_{n}^{*} X_{n}$ for large $n$, where $X_{n}$ is Zariski closed and irreducible of dimension $e \cdot \operatorname{rk}(X)$. So if $Y$ is irreducible, 
and $X$ is a proper subset of $Y$, then (with the parallel notation) $X_{n}$ must be a proper subset of $Y_{n}$, so $e \cdot \operatorname{rk}(X)<e \cdot \operatorname{rk}(Y)$, and $\operatorname{rk}(X)<\operatorname{rk}(Y)$.

It remains to prove the "dimension theorem" 2.3(iii). Let $X, X^{\prime}$ be closed irreducible subsets of $P^{k}, \operatorname{rk} X=d, \operatorname{rk} X^{\prime}=d^{\prime}$, and let $Y$ be a component of $X \cap X^{\prime}$. We must show: $\operatorname{rk} Y \geq d+d^{\prime}-k$. Let the notation $X_{n}, X_{n}^{\prime}$, etc. be as above, and let $n$ be large. Let $Y_{n} \#$ be the component of $X_{n} \cap X_{n}^{\prime}$ containing $\lambda_{n} Y$. We have $\operatorname{dim}\left(X_{n}\right)=d e, \operatorname{dim}\left(X_{n}^{\prime}\right)=d^{\prime} e$. By the dimension theorem for the $k e$-dimensional smooth algebraic variety $P_{n}^{* k}$,

$$
\operatorname{dim} Y_{n} \# \geq d e+d^{\prime} e-k e=e\left(d+d^{\prime}-k\right)
$$

Evidently $r_{n}^{-1} Y_{n} \#=Y_{n+1} \#$. Hence $\operatorname{dim} Y_{n} \#$ is nondecreasing with $n$, so we choose $n$ large enough that $\operatorname{dim} Y_{n} \#=\operatorname{dim} Y_{N} \#$ for $N \geq n$. However, we do not yet know that $\lambda^{n} Y$ is Zariski dense in $Y_{n} \#$. (And this would not be true without thinness.) The problem may arise that for generic $c$ in $Y_{n} \#$, for some $q=p^{l}$ and some polynomials $f_{i}$ over $K, \sum f_{i}(c)^{q} e_{l, i}=0$; in this case, every $F$-point $c^{\prime}$ of $Y_{n} \#$ must also satisfy $f_{i}\left(c^{\prime}\right)=0$ for each $i$, and this may force $c^{\prime}$ to lie on a proper subvariety of $C_{n}$. To rule out this scenario we apply Claim 2 again.

Let $X$ be a set of variables appropriate for describing elements of $P_{n}^{k} ; X=$ $\left(X_{1}, \ldots, X_{k}\right)$ where each $X_{i}=X_{i, 1}, \ldots, X_{i, p^{n}}$. Let $I \#, I, I^{\prime}$ denote the ideals of $K[X]$ vanishing on $Y_{n} \#, X_{n}, X_{n}^{\prime}$ respectively.

Claim 2.12.3. If $\sum f_{i}^{q} e_{l, i} \in I\left(Y_{n} \#\right)$, then $f_{i} \in I\left(Y_{n} \#\right)$ for each $i$.

Proof. I\# is one of the prime components of $\sqrt{ }\left(I+I^{\prime}\right)$, so there exists $h \notin I \#$ and an integer $s$ such that $\left(h\left(\sum f_{i}^{q} e_{l, i}\right)\right)^{s} \in I+I^{\prime}$. By enlarging $s$, we may assume it is a power of $p$. Replacing $q$ by $q s, h$ by $h^{s}$, and $l$ by $l^{\prime}$ (where $p^{l^{\prime}}=q s$ ), we may assume $s=1$ (note that $e_{l, i}^{s}$ are some of the $e_{l^{\prime}, j}$ ). Multiplying by $h^{q-1}$ we have $h^{q}\left(\sum f_{i}^{q} e_{l, i}\right) \in I+I^{\prime}$, so $\sum\left(f_{i} h\right)^{q} e_{l, i} \in I+I^{\prime}$. Now if we show that $f_{i} h \in I \#$ for each $i$, then also $f_{i} \in I \#$, as $I \#$ is a prime ideal. Thus we may take $h=1$. So $\sum f_{i}^{q} e_{l, i} \in I+I^{\prime}$. Say

(i) $\sum f_{i}^{q} e_{l, i}=g+g^{\prime}, g \in I, g^{\prime} \in I^{\prime}$.

At this point we make a change of variables. Let $N=n+l$, and let $r=$ $r_{n} r_{n+1} \cdots r_{N-1}$, so that $\lambda_{n}=r \lambda_{N}$. Let $Y=\left(Y_{1}, \ldots, Y_{p^{N}}\right)$ be a set of variables appropriate for elements of $P_{N}$. Let $r^{*}: K[X] \rightarrow K[Y]$ be dual to $r$; it carries $K[X]$ into $K\left[Y^{q}\right]$. Since $r$ takes $X_{N}$ into $X_{n}, r^{*}$ takes $I$ into $I\left(X_{N}\right)$, and similarly $I^{\prime}$ into $I\left(X_{N}^{\prime}\right)$. Thus $r^{*}(g) \in I\left(X_{N}\right), r^{*}\left(g^{\prime}\right) \in I\left(X_{N}^{\prime}\right)$. We have:

(ii) $\sum r^{*}\left(f_{i}\right)^{q} e_{l, i}=r^{*}(g)+r^{*}\left(g^{\prime}\right)$.

Now we can decompose $r^{*}(g)=\sum e_{l, i} H_{i}$, with $H_{i} \in K^{q}[Y]$. Since $r^{*}(g)$ is in $K\left[Y^{q}\right]$, so is each $H_{i}$; so we may write $r^{*}(g)=\sum e_{l, i} h_{i}^{q}$, with $h_{i} \in K[Y]$. Similarly $r^{*}\left(g^{\prime}\right)=\sum e_{l, i} h_{i}^{\prime q}$. Since $r^{*}(g) \in I\left(X_{N}\right)$, and $\lambda_{N}(X)$ is Zariski dense in $X_{n}$, we have $h_{i} \in I\left(X_{N}\right)$ for each $i$ (as was argued above). Similarly $r^{*}\left(g^{\prime}\right)=\sum e_{l, i} h_{i}^{\prime q}$, with $h_{i}^{\prime} \in I\left(X_{N}^{\prime}\right)$. Now

(iii) $\sum r^{*}\left(f_{i}\right)^{q} e_{l, i}=\sum\left(h_{i}+h_{i}^{\prime}\right)^{q} e_{l, i}$.

Comparing coefficients of each monomial and using the fact that the $e_{l, i}$ are linearly independent over $K^{q}$, this equality of polynomials implies that $r^{*}\left(f_{i}\right)=$ $h_{i}+h_{i}^{\prime}$ for each $i$. Thus $r^{*}\left(f_{i}\right) \in I\left(X_{N}\right)+I\left(X_{N}^{\prime}\right) \subseteq I\left(Y_{N} \#\right)$. But $\operatorname{dim}\left(Y_{N} \#\right)=$ $\operatorname{dim}\left(Y_{n} \#\right)$, and $r$ is generically finite-to-one, so $r Y_{N} \#$ is Zariski dense in $Y_{n} \#$. Thus $r^{*}$ induces a 1-1 map from $K[X] / I\left(Y_{n} \#\right)$ to $K[Y] / I\left(Y_{N} \#\right)$, so $r^{*}\left(f_{i}\right) \in I\left(Y_{N} \#\right)$ implies $f_{i} \in I\left(Y_{n}\right) \#=I \#$. This finishes the proof of the claim. 
It follows from the claim that $\lambda_{n}(Y)$ is Zariski dense in $Y_{n} \#$, so $Y_{n} \#=Y_{n}$, and $\operatorname{rk}(Y) \cdot e=\operatorname{dim}\left(Y_{n}\right)=\operatorname{dim}\left(Y_{n} \#\right) \geq e\left(d+d^{\prime}-k\right)$, so $\operatorname{rk}(Y) \geq d+d^{\prime}-k$, as required by the dimension theorem.

Corollary 2.13. Let $T(p, \nu)$ be the theory of separably closed fields $F$ of char $p>0$, Ersov invariant $\nu<\infty$. Let $P$ be a minimal thin type in $T(p, \nu)$. If $P$ is not locally modular, then $P$ is nonorthogonal to $F^{p^{\infty}}=\bigcap\left\{F^{q}\right.$ : q a power of $\left.p\right\}$.

Proof. By [Wood79] $T(p, \nu)$ is stable. By Lemma 2.5 we may assume $P=\bigcap_{n} P_{n}$, where $P_{n}$ is a subfield of some definable field $K$. By [Mes], 3.6, $K$ is definably isomorphic to a finite extension of $F$, hence is a subfield of $F^{1 / q}$ for some $q$. So we may assume $K$ is a subfield of $F$. By [Mes], 3.1, each $P_{n}$ contains some $F^{q}$. Hence $P$ contains $F^{p^{\infty}}$. Since $P$ is minimal, $P=F^{p^{\infty}}$.

By an $\infty$-definable subgroup of a group $G$ we will mean the intersection of countably many definable subgroups. A result from [Hr90] (mentioned above for fields) states that an $\infty$-definable subset, which is also a subgroup, is an $\infty$-definable subgroup in this sense.

Remark 2.14. The following will emerge in $\S 5$ : Let $F$ be a saturated model of $T(p, \nu)$. Let $\underline{G}$ be a connected algebraic group defined over $F, G=\underline{G}(F)$, and $A$ an $\infty$-definable subgroup of $G$. Assume $A$ has no proper nontrivial definable subgroups, and has thin generic type. Then either $A$ is minimal and locally modular, or $\underline{G}$ is isogenous (as an algebraic group) to a group $\underline{H}$ defined over $F^{p^{\infty}}$, by an isomorphism carrying $A$ to $H\left(F^{p^{\infty}}\right)$.

Let $F$ be a saturated separably closed field. The following lemma shows (using 2.12) that the group of infinitely-divisible points of a commutative algebraic group over $F$ has finite Morley dimension.

Lemma 2.15. Let $\underline{G}$ be a $k$-dimensional commutative algebraic group defined over $F, G=\underline{G}(F)$. Let $A=p^{\infty} G=\bigcap_{n} p^{n} G$. Then any generic type of $A$ is $k$-thin.

Proof. Say $A$ is defined over $K=\operatorname{dcl}(K)$. Let $a \in A$. Given $q=p^{n}$, let $a=q b$ with $b \in G$. By Weil's theorem on symmetric functions, $a \in K\left(b^{q}\right)$ (this is in fact just the content of Lemma 4 in Chapter 1 of [Weil48]). This proves the criterion of Lemma 2.9 .

The following lemmas will inform the ensuing discussion but will not be explicitly used; they are included here in order to clarify the picture.

Lemma 2.16. Let $\underline{G}$ be a simple Abelian variety defined over $F$, but not isomorphic to one defined over $F^{p^{\infty}}$. Let $G=\underline{G}(F)$ and $A=p^{\infty} G$. Then $A$ has no proper infinite definable subgroups.

Proof. We work over a relatively algebraically closed substructure, over which $\underline{G}$ is defined. Let $e=\operatorname{dim}(\underline{G})$. It suffices to show that $A$ is contained in every infinite definable subgroup of $G$. Let $H$ be such a definable subgroup. Let $G_{n}=\lambda_{n} G$, and let $\underline{H}_{n}$ be the Zariski closure of $\lambda_{n} H$. Then for large enough $n, H=G \cap \lambda_{n}^{-1} \underline{H}_{n}(F)$. We will say something about $F, G, A$, and $H$ in turn.

Let $K=F^{p^{-n}}$. Then $K=F\left[e_{1}^{p^{-n}}, \ldots, e_{\nu}^{p^{-n}}\right]$. Let $\underline{F}$ be the algebraic closure of $F$, and let $\underline{R}$ be the ring $\underline{F}\left[e_{1}^{p^{-n}}, \ldots, e_{\nu}^{p^{-n}}\right]$. Then $\underline{R}$ can be viewed as an affine algebraic ring, and $K$ as the set of $F$-rational points of $\underline{R}$. Note that $\underline{R}$ is isomorphic, over $\underline{F}$, to $\underline{F}\left[u_{1}, \ldots, u_{\nu}\right]$, where the $u_{i}$ are commuting infinitesimals: $u_{i}^{p^{n}}=0$. 
By taking $p^{n}$-th roots of points of $\lambda_{n} G$, we identify $\lambda_{n} G$ with the set of $K$ points of $\underline{G}_{1}=\operatorname{Fr}^{-n}(\underline{G})$. Viewing $K$ as $\underline{R}(F)$ as above, we see that $\lambda_{n} G$ can be identified with $\underline{G}_{1}(\underline{R}(F))$. Now the "composition" $\underline{G}_{2}=\underline{G}_{1}(\underline{R})$ is another group scheme, and since $\underline{R}(\underline{F})$ is isomorphic to $\underline{F}\left[u_{1}, \ldots, u_{\nu}\right], \underline{G}_{2}$ is an extension of $\underline{G}_{1}$ by a commutative unipotent group $\underline{G}_{u}$. Let $\underline{G}_{3}$ be the closed subgroup of $p^{\infty} \underline{G}_{2}$. Then $\underline{G}_{3}$ meets $\underline{G}_{u}$ in a finite group. Hence $\underline{G}_{3}$ is isogenous to $\underline{G}_{1}$ (over $\underline{F}$ ). So $\underline{G}_{3}$ is a simple Abelian variety of dimension $e$.

Let $\underline{A}_{n}$ be the Zariski closure of $\lambda_{n}(A)$. Then $\lambda_{n}(A) \subseteq p \underline{A}_{n}$, so $p \underline{A}_{n}=\underline{A}_{n}$, and hence $\underline{A}_{n} \subseteq p^{\infty} \underline{G}_{2}(\underline{F})=\underline{G}_{3}(\underline{F})$. Since $\underline{G}_{3}$ is simple, $\underline{A}_{n}=\underline{G}_{3}$.

It follows that $\underline{H}_{n} \cap \underline{A}_{n}$ is finite, or else $\underline{H}_{n}$ contains $\underline{A}_{n}$. In the first case, $H$ contains only finitely many $p^{n}$-th powers. In particular $H^{p^{n}}$ is finite. Since the $p^{n}$-torsion points of $\underline{G}$ are finite in number, $H$ is finite; a contradiction. So $\underline{H}_{n}$ contains $\underline{A}_{n}$, hence $H=G \cap \lambda_{n}^{-1} \underline{H}_{n}(F)$ contains $A$.

Notation. $G_{[q]}=\{x \in G: q x=0\}$.

Lemma 2.17. Let $\underline{G}$ be a commutative algebraic group, and $A$ an $\infty$-definable subgroup of $G=\underline{G}(F)$ of finite Morley dimension, or just: with no properly descending sequence of definable subgroups. Then $A \subseteq p^{\infty} G+G_{\left[p^{n}\right]}$ for some $n$.

Proof. The chain of subgroups $A \cap p^{n} G$ must stabilize. So $A \cap p^{n} G=A \cap p^{\infty} G$ for some $n$. Hence if $a \in A$, then $p^{n} a \in p^{\infty} G$. Using saturation of $F, p^{\infty} G$ is $p$-divisible; so $p^{n} a=p^{n} b$ for some $b \in p^{\infty} G$. Thus $p^{n}(a-b)=0$, so $a-b \in G_{\left[p^{n}\right]}$, and $a=b+(a-b)$.

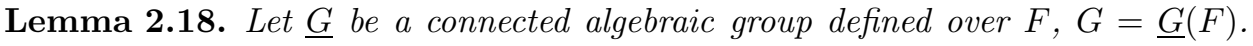
Then $G$ is connected.

Proof. Let $H$ be a definable subgroup of $G$ of finite index. Let $G_{n}=\lambda_{n} G$; then $G_{n}$ can be endowed with a group structure in such a way that $\lambda_{n}$ is an isomorphism; and $G_{n}=\underline{G}_{n}(F)$, where $\underline{G}_{n}$ is an algebraic group, isomorphic over $F^{a}$ to a power of $\underline{G}$. Let $\underline{H}_{n}$ be the Zariski closure of $\lambda_{n} H$. Then for large enough $n, H=G \cap \lambda_{n}^{-1} \underline{H}_{n}(F)$. $\lambda_{n} H$ has finite index in $\lambda_{n} G$, hence $\underline{H}_{n}$ has finite index in $\underline{G}_{n}$. But $\underline{G}_{n}$ is connected; so $\underline{H}_{n}=\underline{G}_{n}$, and $H=G$.

Remark 2.19. Let $\underline{G}$ be a simple Abelian variety, defined over $F$, not isomorphic to one defined over $F^{p^{\infty}}, G=G(F)$. Let $A=p^{\infty} G$. In 2.15 we showed that $A$ has finite Morley dimension, and in 2.16 that $A$ is minimal as a group. This suggests that $\operatorname{dim}(A)=1$. This will indeed be shown in $\S 5$ as a consequence of modularity; we do not know a direct proof.

2.20 Guide to $\S \mathbf{6}$ of $[\mathrm{HZ}]$. This is intended for the reader who wishes to obtain 2.5 as efficiently as possible, reading only $\S 6$ of [HZ] and one preceding page. $\S 6.1$ is motivation and includes no results. $\S 6.2$ is written for minimal types of stable theories, and can be read directly. In $\S 6.3$ one assumes in addition a notion of specialization and of a regular specialization between tuples of elements of the minimal type, satisfying certain axioms. We will immediately give a definition of these that may be used when $D$ is a thin type in a separably closed field. In Lemmas 6.8 and 6.10 , using $§ 6.2$, one obtains an Abelian group of dimension one. Then 6.9 and 6.11 work with the group elements and provide the required field.

The group obtained is again a thin type of $U$-rank one in a separably closed field, and so the same definitions and axioms of specialization may be used. The 
dimension theorem 2.3(iii) has been proved only for complete types $P$. However, if $D$ is an $\infty$-definable group of dimension one, one may choose a complete type $P$ contained in $D$, and a generic element $a$ of $P$; then $D$ is covered by $P$ and $(P+a)$, translation by $a$ gives a homeomorphism in every dimension, and the dimension theorem thus holds in $P$ and $P+a$ and hence in $D$.

It remains to define the notion of specialization and regular specialization, and prove the axioms used in $\S 6.3$ of [HZ]. Let $D$ be a thin type in a separably closed field; we suppose $D$ is either a complete type over some base set, or else an $\infty$ definable group of dimension one. The notion of closed set used in this section gives rise to one specialization: $a \rightarrow b$ if $b$ lies in every 0-definable closed set in which $a$ lies. We can also describe this directly. Given an element $a$ of $D^{n}$, write $\lambda a$ for the sequence $\left(a, \lambda_{1} a, \lambda_{2} a, \ldots\right)$. We write $a \rightarrow b$ if $\lambda a$ specializes to $\lambda b$ in the ordinary field-theoretic sense. In other words, any polynomial with coefficients in the base field vanishing on any $\lambda_{n} a$, also vanishes on $\lambda_{n} b$; or again, $\lambda_{n} b$ is an element of the locus of $\lambda_{n} a$. We say that the specialization is regular if for each $n, \lambda_{n} b$ is a nonsingular point on the locus of $\lambda_{n} a$.

We now indicate the proof of the axioms 6.6 of [HZ].

(1) is trivial.

(2) follows from the same fact in algebraically closed fields, applied to $\lambda a, \lambda b$.

(3) This follows from 2.3(iii): $a^{\prime}$ is any generic point of the component of the intersection of $\operatorname{locus}(a)$ with the diagonal $x(1)=x(2)$ containing the locus of $a^{\prime \prime}$.

(4) and (5) require reading the page preceding $\S 6$, starting with the definition of "good" specialization. The amalgamation Lemma 5.14 in [HZ] for regular specializations follows from the same lemma in the case of algebraically closed fields, applied to $\lambda a, \lambda b$, etc. Observe that amalgamating two fields over a ( $\lambda$-closed) substructure of a separably closed field can never create inseparability. Now follow the proof of 5.14 for good specializations, and of 5.15 in [HZ]

(6) This states that the graph of addition is closed; indeed by results from [Hr90] or [Mes], addition can be taken to be given locally by rational functions.

(7) The first statement follows from the fact that a product of smooth varieties is smooth. For the second let $a$ be a generic point of $D$ and $a^{\prime}$ any point of $D$; we need to know that $\lambda_{n} a^{\prime}$ is a nonsingular point of the locus of $a$. In case $D$ is a complete type, $a^{\prime}$ must also be generic, so this is trivial. In case $D$ carries a group structure, all points on the locus are smooth.

\section{MANIN'S HOMOMORPHISM AND BUIUM'S REDUCTION}

In this section we show that the main Theorem follows from a slightly different version, in which $\Gamma$ is replaced by a certain definable subgroup. For Abelian varieties in characteristic 0 , this was observed by Buium, and forms the basis of his approach in [Bu92], [Bu93].

We work in a universal domain $F$ for differential fields of characteristic 0 , or for fields with a distinguished $p$-basis $\left\{e_{i}\right\}_{i=1, \ldots, p^{\nu}}$. Definable or $\infty$-definable sets are understood in the sense of $F$, as is Morley dimension, etc.

We will eventually prove:

Theorem 5.9. Let $K$ be either a separably closed field of characteristic $p>0$, with a finite $p$-basis fixed, or a differentially closed field of char 0 . Let $k=\bigcap_{n} K^{p^{n}}$ if $p>0, k=\{x: D x=0\}$ if the characteristic is 0 . Let $\underline{S}$ be a semi-Abelian variety defined over $K, \underline{X}$ a subvariety, $S=\underline{S}(K), X=\underline{X}(K)$, and let $\Gamma_{n}(n=1,2, \ldots)$ 
be a descending sequence of definable subgroups of $S$, such that $\bigcap_{n} \Gamma_{n}(F)$ has finite dimension. Assume that for each $n$, for some coset $C_{n}$ of $\Gamma_{n}, X \cap C_{n}$ is Zariski dense in $\underline{X}$. Then there exists a semi-Abelian variety $\underline{S}_{0}$ defined over $k$, a subvariety $\underline{X}_{0}$ of $\underline{S}_{0}$ defined over $k$, and a rational homomorphism $h$ from a group subvariety of $\underline{S}$ into $\underline{S}_{0}$, such that $\underline{X}=h^{-1}\left(\underline{X}_{0}\right)+c$ for some $c$.

We now argue that this is sufficient. Let $S, X, k, \Gamma$ be as in 1.1. We choose a finitely generated field extension $L$ of $k$, such that $S, X$ and some $p^{\prime}$-generators of $\Gamma$ are defined over $L$. In characteristic $p,\left[L: L^{p}\right]$ is finite, and $\bigcap_{n} L^{p^{n}}=k$. The same remains true for the separable closure $K$ of $L$. In characteristic 0 , we endow $L$ with a differential structure over $k$ such that $k$ is the field of constants, and we let $K$ be a differential closure. It remains only to replace $\Gamma$ by an appropriate definable subgroup.

In characteristic 0 we use:

Lemma 3.1. Let $S$ be a semi-Abelian variety defined over $F$. Let $\Gamma$ be a $p^{\prime}$-finitelygenerated subgroup of $S$. Then there exists a subgroup of $S$ of finite dimension containing $\Gamma$.

Proof. This is proved in [Bu93] for Abelian varieties; the proof goes through in the semi-Abelian case.

In characteristic $p$, we let $\Gamma_{n}=p^{n} S(K)$. The fact that the intersection of the groups $\Gamma_{n}$ has finite dimension was shown in the previous section. If $\Gamma$ is a $p^{\prime}$ finitely generated subgroup of $S(K)$, then $\left(\Gamma / p^{n} \Gamma\right)$ is finite, so $\Gamma$ meets only finitely many cosets of $\Gamma_{n}$. Hence some coset of $\Gamma_{n}$ meets $X$ in a Zariski dense set.

In either case, 1.1 follows from 5.9.

\section{Abelian groups of Finite Morley dimension}

We work in this section with the category of $\infty$-definable groups and morphisms within some saturated stable structure $\mathbb{C}$. All groups are assumed to have finite Morley dimension.

A group $G$ of finite Morley dimension always has a maximal connected semi-pluriminimal subgroup $S_{1}(G)$. For algebraic groups, we always have $S_{1}(G)=G^{0}$ (the connected component). In general this fails however. In Proposition 4.3 we show how to reduce certain questions about definable subsets of $G$ to similar questions in $S_{1}(G)$ and in proper quotients of $G$; for example, a definable subset of $G$ containing no cosets of infinite subgroups of $G$ is contained in finitely many cosets of $S_{1}(G)$. We then proceed to analyze semi-pluriminimal groups; they are an "almost" direct sum of pairwise orthogonal definable subgroups, each of which is semi-minimal.

Certain basic notions of algebraic groups generalize to the present context; one must give definitions that do not rely on the Zariski topology, but only on dimension theory for constructible sets. The definitions and facts in 4.0 are due to Zilber and Poizat; see [NeP89].

Definition 4.0. (a) An $\infty$-definable group $G$ is connected if it has no definable subgroups of finite index.

(b) Let $G$ be an $\infty$-definable group, and $X$ a definable subset of $G$. The stabilizer $\operatorname{Stab}(X)$ is

$$
\{g \in G: \operatorname{dim}((X-g X) \cup(g X-X))<\operatorname{dim}(X)\} .
$$

It is a definable subset of $G$. 
(c) An $\infty$-definable subset $X$ of $G$ is indecomposable if whenever $X$ is contained in a finite union of cosets of a definable subgroup $H$ of $G$, it is contained in a single coset. If $X$ is a complete type of degree 1 , then it is indecomposable. If $X$ is indecomposable, then the coset generated by it is generated in finitely many steps.

Definition 4.1. Let $G$ be an Abelian group, $\infty$-definable over a base set $B$, and $X$ a definable set over $B . G$ is $X$-rigid if every connected definable subgroup of $G$ can be defined over a set $C$ independent from $X(\mathbb{C})$ over $B$. $G$ is rigid if every connected definable subgroup is defined over $\operatorname{acl}(B)$. Equivalently, every connected definable subgroup is defined over some fixed countable set. $G$ is strongly rigid if the same holds for arbitrary (not necessarily connected) subgroups.

Definition 4.2 (of "full" orthogonality of two $\infty$-definable sets $X, Y$ ). $X, Y$ are orthogonal if for any algebraically closed $B \subseteq \mathbf{C}^{\text {eq }}$ with $X, Y$ defined over $B$, any $b \in X$ and any $c \in Y, \operatorname{tp}(b / B) \cup \operatorname{tp}(c / B)$ implies $\operatorname{tp}(b c / B)$.

In our applications of 4.3 below, $A$ will be the maximal connected semi-pluristrongly minimal subgroup of $G$, and will be strongly rigid.

Proposition 4.3. Let $G$ be an Abelian group of finite Morley dimension, $A$ a nontrivial connected definable subgroup, and $X$ an $(\infty-)$ definable subset of $G$ of multiplicity 1. Assume:

(i) $A$ is $G / A$-rigid.

(ii) There is no definable group $A^{\prime} \supset A$ with $A^{\prime} / A$ infinite and $A^{\prime} \subseteq \operatorname{acl}(Y, A, C)$ for some minimal $Y$ and finite $C$.

(iii) $\operatorname{Stab}(X) \cap A$ is finite.

Then $X$ is contained in a single coset of $A$, up to a set of smaller dimension.

Proof. We may assume $G, A, X$ are 0 -definable. It is convenient to replace $X$ by the corresponding complete type, i.e. to remove from $X$ all 0-definable subsets of $X$ of smaller rank. So we must now show that $X$ is contained in a single coset of $A$. Let $\theta: G \rightarrow G / A$ be the canonical homomorphism, and let $X / A=\theta(X)$. For $b \in(X / A)$, let $A(b)=\theta^{-1}(b)$, a coset of $A$.

Let $b$ be an element of $X / A$. Then $X \cap A(b) \neq \emptyset$. Let $U$ be a nonempty subset of $A(b)$, defined over $\{b\} \cup A$, of least possible dimension and multiplicity. For $c \in A$, we can consider the translate $U+c$ of $U$ within $A(b)$. Then $(U+c) \cap U$ is defined over $\{b\} \cup A$. Necessarily either $U \cap(U+c)$ or $U-(U+c)$ has smaller dimension or multiplicity than $U$; so one of them is empty. It follows that $U$ is a coset of some subgroup $K$ of $A$ (namely $K=\{c \in A: U=U+c\}$ ). By considering intersections of $U$ with $A$-translates of $X$, we see that every $A$-translate of $U$ meets $X$ trivially or is contained in $X$; so $X \cap A(b)$ is $K$-invariant.

By the rigidity assumption, $K^{o}$ is defined over a set $F_{0}$ orthogonal to $G / A$; so $b$ remains a generic element of $X / A$ over $F_{0}$. Thus $X \cap A\left(b^{\prime}\right)$ is invariant under translation by $K^{o}$, for generic $b^{\prime} \in(X / A)$. So $K^{o} \subseteq \operatorname{Stab}(X)$. By (iii), $K$ is finite.

Since $U$ is a coset of $K$, it is also finite. Recalling that $U$ is defined over $\{b\} \cup A$, we have $U \subseteq \operatorname{acl}(b, A)$. Every element of $A(b)$ has the form $a+x$ for some $a \in A$, $x \in U$, so $A(b) \subseteq \operatorname{acl}(b, A)$.

If $\operatorname{rk}\left(b / F_{0}\right)=0$, then $X / A$ is finite, and having multiplicity 1 , it consists of a single element; in other words $X$ is contained in a single coset. Otherwise, we will get a contradiction. Increase $F_{0}$ to $F_{1}$ so that $\operatorname{rk}\left(b / F_{1}\right)=1$, and let $Y$ be the locus of $b$ over $F_{1}$, and $X^{\prime}=\{x \in X: x+A \in Y\}$. Then $A(b) \subseteq \operatorname{acl}(b, A)$ for $b \in Y$, so $X^{\prime} \subseteq \operatorname{acl}(Y \cup A)$. By the indecomposability theorem, for some finite 
$m,\left\{\sum n_{i} y_{i}:\left(y_{1}, \ldots, y_{m}\right) \in Y^{m},\left(n_{1}, \ldots, n_{m}\right) \in \mathbf{Z}^{m}, \sum_{i} n_{i}=0\right\}$ is a subgroup of $G / A$. So $\left\{a+\sum n_{i} b_{i}: a \in A,\left(b_{1}, \ldots, b_{m}\right) \in X^{\prime m},\left(n_{1}, \ldots, n_{m}\right) \in \mathbf{Z}^{m}, \sum_{i} n_{i}=0\right\}$ is a subgroup of $G$, and evidently it contains $A$ and is contained in $\operatorname{dcl}\left(A \cup X^{\prime}\right) \subseteq$ $\operatorname{acl}(Y \cup A)$. This contradicts assumption (ii).

Various formulations of this proposition lift easily to the superstable context. We include a variation using full orthogonality, though it will not be used for the proof of the main theorem.

Proposition 4.4. Let $G$ be an Abelian group of finite Morley dimension, $X$ a definable subset of $G$, and $A$ a connected definable subgroup. Assume $A$ is orthogonal to $G / A$. Then $X$ is a finite union of definable subsets $X_{i}$ with the following property: For some definable subgroup $H_{i}$ of $G, X_{i}$ is a union of cosets of $\left(H_{i} \cap A\right)^{\circ}$, and is contained in a single coset of $H_{i}+A$.

Proof. Let $G^{*}=G / A$. Each element $b \in G^{*}$ can be thought of as a coset of $A$ in $G$, which we denote as $A(b)$. There is no loss of generality in assuming $\operatorname{acl}^{\mathrm{eq}}(\emptyset)=\mathrm{dcl}^{\mathrm{eq}}(\emptyset)$, i.e. all types over $\emptyset$ are stationary. Let $X \# \subseteq G^{*}$ be the solution set of a complete type over $\operatorname{acl}^{\mathrm{eq}}(\emptyset)$. We will find a 0 -definable subgroup $H$ of $G$ such that $\{x \in X: x+A \in X \#\}$ is contained in a coset of $H+A$, and is a union of cosets of $(H \cap A)^{\circ}$. By compactness, there exists a 0-definable set $X^{*}$ containing $X \#$ such that $\left\{x \in X: x+A \in X^{*}\right\}$ is contained in a coset of $H+A$ (and of course this set is still closed under translation by elements of $\left.(H \cap A)^{\circ}\right)$. Thus $G^{*}$ can be covered with 0 -definable sets with this property. The statement of the proposition follows by another application of compactness.

Pick $b \in X \#$, and also $g \in A(b)$. Let $S=\{c \in A: g+c \in X\}$. By stable definability, $S$ is definable with parameters $C$ from $A$. We have

(*) For some $g \in A(b), A(b) \cap X=S+g$.

By the orthogonality assumption, $\operatorname{tp}\left(b^{\prime} / C\right)$ does not depend on $b^{\prime} \in X \#$. Thus $(*)$ holds for any $b \in X^{\#}$. Let $K=\{c \in A: c+S=S\}$. $K$ is a definable subgroup of $A$. Now if $S+g=S+g^{\prime}$, then $g-g^{\prime} \in K$. Thus we have a definable map $f: X \# \rightarrow G / K$ (given by: $f(b)=g+K$ iff $A(b) \cap X=S+g$ ). $f$ is a section of the natural projection $\pi:(G / K) \rightarrow(G / A)$.

Claim. Let $C^{*}$ be the coset generated by $X \#$ in $G^{*}$. Then $f$ extends to an affine homomorphism from $C^{*}$ to $G$.

Proof. For large enough odd $n$, and $\left(a_{1}, \ldots, a_{n}\right) \in X \#^{n}$ generic, $\sum(-1)^{i} a_{i}$ is a generic element of the connected, definable coset $C^{*}$ ("indecomposability theorem"). It suffices to show that for all $n$ there exists a constant $\chi_{n}$ such that for all generic $\left(a_{1}, \ldots, a_{n}\right) \in X \#^{n}, f\left(\sum^{\prime} a_{i}\right)-\sum^{\prime} f\left(a_{i}\right)=\chi_{n}$, where $\sum^{\prime}$ denotes the alternating sum. Let $h\left(a_{1}, \ldots, a_{n}\right)=f\left(\sum^{\prime} a_{i}\right)-\sum^{\prime} f\left(a_{i}\right)$. Then since $f$ is a section of $\pi, h\left(a_{1}, \ldots, a_{n}\right)=0(\bmod A / K)$, i.e. $h\left(a_{1}, \ldots, a_{n}\right) \in A / K$. Thus $h$ is a map from $X \#^{n}$ into $A / K$; since they are orthogonal, $h$ is generically constant.

Thus there exists a connected definable subgroup $H^{*}$ of $G^{*}$ and a nontrivial definable group homomorphism $h: H^{*} \rightarrow G / K$. Let $H$ be a subgroup of $G$ containing $K$, such that $H / K=h H^{*}$. Note that $(H+A) / A=H^{*}$.

Claim. $(H \cap A)^{\circ} \subseteq K$.

Proof. Clearly $H \cap A \supseteq K$. We must show that $(H \cap A) / K$ is finite. The homomorphism $h$ induces an isomorphism between $(H \cap A) / K$ and $h^{-1}((H \cap A) / K) / \operatorname{Ker}(h)$, a quotient of two definable subgroups of $G^{*}$. By the orthogonality assumption, both sides must be finite.

This finishes the proof. 
Definition 4.5. If $A$ is a definable group and $X$ a minimal set, then there exists a unique maximal connected subgroup $C$ of $A$ such that $C \subseteq \operatorname{acl}(X, F)$ for some finite $F$. We denote this group by $A\langle X\rangle$.

Lemma 4.6. Suppose $A$ is connected, and $A \subseteq \operatorname{acl}(Y)$ for some pluriminimal $Y$. Then $A$ is isogenous to a direct sum of pairwise orthogonal semi-minimal groups (namely to the direct sum of the various nontrivial subgroups $A\langle X\rangle, X$ minimal).

Proof. Clearly if $X$ is orthogonal to $X_{1} \cup \cdots \cup X_{k}$, then

$$
A\langle X\rangle \cap\left(A\left\langle X_{1}\right\rangle+\cdots+A\left\langle X_{k}\right\rangle\right)
$$

is finite. If $X_{i}, X_{j}$ are nonorthogonal, then $A\left\langle X_{i}\right\rangle=A\left\langle X_{j}\right\rangle$. Thus the group $B$ generated by all $A\langle X\rangle$ (X minimal) is a finite orthogonal sum. We must show $B=$ $A$. Let $c$ be a generic element of $A, b=c+B \in A / B$. We have $c \in \operatorname{acl}\left(y_{1}, \ldots, y_{k}\right)$ for some $y_{j}$ from $Y$, hence $c \in \operatorname{acl}\left(b, y_{1}, \ldots, y_{k}\right)$. Otherwise, minimizing $k$, we may assume $y_{j} \notin \operatorname{acl}\left(b, y_{1}, \ldots, y_{j-1}\right)$ for each $j$. So $\left\{b, y_{1}, \ldots, y_{k}\right\}$ is an independent set. In particular, $c \notin \operatorname{acl}\left(y_{1}, \ldots, y_{k}\right)$.

Let $Z$ be the locus of $c$ over $\left\{y_{1}, \ldots, y_{k}\right\}$. Then $Z$ is infinite, but any element $c$ of $Z$ is algebraic over $c+B$. Replace $Z$ by a minimal $\infty$-definable subset $Z^{\prime}$; it has the same property. $A\left\langle Z^{\prime}\right\rangle$ is nontrivial, so it is contained in $B$. But $Z^{\prime} / A\left\langle Z^{\prime}\right\rangle$ must be finite, while $Z^{\prime} / B$ is infinite, a contradiction.

Lemma 4.7. Suppose $A$ is connected, semi-minimal, and locally modular. Then $A$ is isogenous to a direct sum of minimal subgroups.

Proof. Let $\left\{A_{i}\right\}$ be a maximal set of minimal subgroups of $A$, such that the map from $\bigoplus_{i} A_{i}$ to $A$ has finite kernel. Let $B$ be the image of this map, and suppose for contradiction that $B \neq A$. As in 4.6 one finds a minimal $Z \subseteq A$ such that $Z / B$ is infinite. But by [HP86] $Z$ is a coset of a definable subgroup $C$ of $A$ (up to a finite number of points). $C$ must be minimal, and $(C+B) / B$ is infinite, a contradiction.

The following lemma is a special case of results from [Hr90]; we include a proof for the reader's convenience.

Lemma 4.8. Let $A$ be a semi-minimal group. Suppose $A$ is nonorthogonal to an $\infty$-definable set $D$. Then there exists a group $B$ with $B \subseteq \operatorname{dcl}(D)$ and a definable surjective homomorphism $h: A \rightarrow B$, with finite kernel.

Proof. We have $A \subseteq \operatorname{acl}(Y)$ for some minimal $Y ; Y$ is necessarily nonorthogonal to $D$, so $Y \subseteq \operatorname{acl}(F, D)$ for some finite $F$, and hence $A \subseteq \operatorname{acl}(F, D)$. Let $a$ be a generic point of $A$ over $F$, and let $\phi(x, y)$ be a formula over $F$ such that $\phi(a, d)$ holds for some $d \in D^{n}$, and for any $d^{\prime}, \phi\left(x, d^{\prime}\right)$ has only finitely many solutions. Let $C(a)=\left\{d \in D^{n}: \phi(a, d)\right\}$. By stability, $C(a)$ can be defined with parameters from $D$; let $b$ be a canonical parameter for $C(a)$. Then $b=g(a)$ for some $F$-definable function $g$. We may assume $g(x) \in \operatorname{dcl}(D)$ for all $x$.

Let $K=\{x \in A$ : for generic $a \in A, g(a+x)=g(a)\}$. Let $a_{0}, \ldots, a_{2 r}$ be mutually independent generic elements of $A, r=\operatorname{rk}(A)$. If $x-y \notin K$, then since some $a_{i}$ is generic over $x, y, g\left(x+a_{i}\right) \neq g\left(y+a_{i}\right)$. Thus the function $f(x)=$ $\left(g\left(x+a_{0}\right), \ldots, g\left(x+a_{2 r}\right)\right)$ is 1-1 modulo $K$. The image $B$ of $A$ by $f$ is contained in $\operatorname{dcl}(A)$, and may be endowed with a group structure so that $f$ is an isomorphism. By stability (cf. [NeP89]), $B$ and its group structure are definable with parameters from $D$. 
Lemma 4.9. Assume: Whenever $H_{1} \subset H_{2}$ are definable subgroups of $G$, there exists a nonzero element of $H_{2} / H_{1}$ algebraic over any base of definition for $H_{1}$. Then $G$ is strongly rigid.

Proof. We assume $G$ is defined over acl $(\emptyset)$, and show every definable subgroup of $G$ is defined over $\operatorname{acl}(\emptyset)$. Let $H(a)$ be an $a$-definable subgroup. Let $H_{1}$ be the intersection of $H\left(a^{\prime}\right)$ over all $a^{\prime}$ realizing $\operatorname{tp}(a / \operatorname{acl}(\emptyset))$. If $H_{1}=H(a)$ we are done. Otherwise there exists a nonzero $b \in H(a) / H_{1}$ with $b$ algebraic over $\emptyset$. Necessarily $b \in H\left(a^{\prime}\right)$ for all $a^{\prime}$, a contradiction.

The following lemma is well known.

Lemma 4.10. Let $k$ be an algebraically closed field and $G$ a semi-Abelian variety defined over $k$. Then $G(k)$ is strongly rigid.

Proof. All torsion points are algebraic, and 4.9 applies.

Lemma 4.11. Let $A$ be a locally modular group. Then $A$ is rigid.

Proof. [HP86].

Lemma 4.12. Let $A, B$ be orthogonal subgroups of a group $G$, and let $X \subseteq(A+B)$ be the solution set of a complete type over $\operatorname{acl}(\emptyset)$. Then $X$ has the form $U+V$, $U \subseteq A, V \subseteq B$.

Proof. Let $(a, b)$ be a point of $X$. Let $U$ be the locus of $a$ and $V$ the locus of $b$. Then by definition of orthogonality, for any $a^{\prime} \in U$ and $b^{\prime} \in V,\left(a^{\prime}, b^{\prime}\right)$ has the same type as $(a, b)$; so $U+V \subseteq X$.

Lemma 4.13. Suppose $A, B$ are orthogonal (strongly) rigid groups. Then their product is (strongly) rigid.

Proof. Applying 4.12 to the generic type, a connected definable subgroup of $A \times B$ is a product of definable subgroups of $A$ and of $B$. Thus an arbitrary definable subgroup of $A \times B$ lies between two definable subgroups $C \subseteq D$ with $[D: C]$ finite, and $C, D$ each a product of subgroups of $A$ and of $B$.

Lemma 4.14. Suppose $F$ is a finite subgroup of $A$, and $A / F$ is (strongly) rigid. Then $A$ is (strongly) rigid.

Proof. Let $H(a)$ be a definable subgroup of $A$. Then $J=H(a)+F$ does not depend on $a$ (if $\operatorname{tp}(a / \operatorname{acl}(\emptyset))$ is given). Hence $H(a)$ is a subgroup of $J$ containing the connected component $H$ of $J$. But $H$ has finite index in $J$, so there are only finitely many intermediate subgroups.

Lemma 4.15. Let $A=A_{1}+A_{2}$, where $A_{1}, A_{2}$ are orthogonal semi-minimal subgroups, and $A_{1}$ is of linear type. Let $X \subseteq A$ be the solution set of a complete type over $\operatorname{acl}(\emptyset)$. Assume $\operatorname{Stab}(X)$ is finite. Then $X$ is contained in a coset of $A_{2}$.

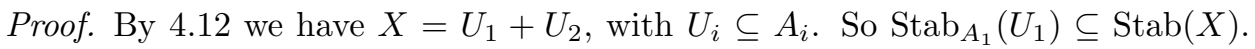
Since $A_{1}$ is locally modular, $U_{1}$ is contained in a coset of $\operatorname{Stab}\left(U_{1}\right)$; so $U_{1}$ is finite, and being the solution set of a complete type, it has one point. Thus $X$ is a translate of $U_{2}$. 


\section{THE MAIN THEOREM}

$K$ is either a separably closed field of characteristic $p>0$, with a finite $p$ basis fixed, or a differentially closed field of char 0 . In either case $K$ is assumed saturated (i.e. we work in the universal domain). Let $k=\bigcap_{n} K^{p^{n}}$ if $p>0$, and $k=\{x: D x=0\}$ if the characteristic is 0 . In either case $k$ is an algebraically closed field. We will say that a type has finite dimension if it has finite Morley dimension and, when $p>0$, is thin.

Our goal is the following version of the main theorem.

Theorem 5.1. Assume $K$ is saturated. Let $S$ be a semi-Abelian variety defined over $K$, and $X$ a subvariety of $S$. Let $\Gamma$ be an $\infty$-definable subgroup of $S$, of finite dimension. Assume $X \cap \Gamma$ is Zariski dense in $X$. Then there exists a semi-Abelian variety $S_{0}$ defined over $k$, a subvariety $X_{0}$ of $S_{0}$ defined over $k$, and a rational homomorphism $h$ from a group subvariety of $S$ into $S_{0}$, such that $X=h^{-1}\left(X_{0}\right)+c$ for some $c$.

Definition. We call a function $h p$-rational if $p=0$ and it is rational, or when $p>0$ if it is the composition of a rational function with some negative power of Frobenius.

Fact 5.2. If $S \subseteq k^{n}$ is a (relatively) definable subset, then $S$ is constructible (i.e. definable in the field structure $(k,+, \cdot)$ with parameters). If $h$ is a definable map on $S$, then $S$ may be split into finitely many constructible sets, on each of which $h$ is a $p$-rational function.

Proof. This follows from quantifier elimination, and the fact that every automorphism of a (differential) field extends to its separable (resp. differential) closure.

Fact 5.3. Let $L$ be an $\infty$-definable field with minimal generic type. Then $L$ is definably isomorphic to $k$.

Proof. This is part of the theses of Sokolovic [So92] when $p=0$, and of Messmer [Mes] when $p>0$.

Lemma 5.4. Let $X$ be a Zariski minimal type. Then either $X$ is locally modular, or $X$ is nonorthogonal to $k$.

Proof. When $X$ is strongly minimal, i.e. it is the solution set of a single formula, it is proved in [HZ] that there exists a field $L$ satisfying the hypotheses of 5.3 , and nonorthogonal to $X$. The proof goes through in the general case. By 5.3, we may take $L=k$.

Lemma 5.5. Let $A$ be a semi-minimal group. Then either $A$ is locally modular, or there exists an algebraic group $\underline{H}$ defined over $k$ and a definable surjective group homomorphism $h: A \rightarrow \underline{H}(k)$, with finite kernel.

Proof. By 5.4, if $A$ is not locally modular, it is nonorthogonal to $k$. By 4.8 , there exists a definable surjective group homomorphism $h$ with finite kernel, such that $B=h A \subseteq \operatorname{dcl}(k)$. By Fact $5.2, B$ is definable in the structure $(k,+, \cdot)$. By [NeP89] (see chapter on Weil's theorem), there exists an algebraic group $\underline{H}$ defined over $k$ such that $B$ is definably isomorphic to $\underline{H}(k)$.

Proposition 5.6. Let $\underline{G}$ be a semi-Abelian variety defined over $K$. Let $G=\underline{G}(K)$. Let $A$ be a semi-minimal definable subgroup of $G$, Zariski dense in $\underline{G}$. Then either 
$A$ is locally modular, or there exists an algebraic group $\underline{H}$ defined over $k$ and $a$ bijective rational homomorphism $\underline{h}: \underline{G} \rightarrow \underline{H}$, with $\underline{h}(A)=\underline{H}(k)$.

Proof. Suppose $A$ is not locally modular. Then by 5.5 there exists an algebraic group $\underline{H}$ defined over $k$ and a definable surjective group homomorphism $h: A \rightarrow$ $\underline{H}(k)$, with finite kernel of size $n$, say. Consider $R=\{(n x, y): h(x)=y\}$. If $(u, 0) \in R$, then $u=n x, h(x)=0$, so $x$ is in the kernel and $u=0$. Thus $R$ defines a homomorphism $g$ from $h(A)$ to $G$. If $(0, y) \in \underline{R}$, then $y$ is the image of an $n$-torsion point of $A$; so $g$ has finite kernel, and is surjective.

By $5.2, g$ is given by a $p$-rational map $g$. Since $h(A)$ is Zariski dense in $\underline{H}, g$ defines a homomorphism $\underline{g}$ on $\underline{H}$ into $\underline{G}$. Let $R$ be the smallest closed subgroup of $H$ with $H / R$ semi-Abelian; then $R$ is defined over $k ; H / R$ is strongly rigid by 4.10 , so $\operatorname{Ker}(\underline{g}) / R$ is defined over $k$; hence $\operatorname{Ker}(\underline{g})$ is defined over $k$. Since $k$ is algebraically closed, there exists an algebraic group $\underline{H}^{*}$ defined over $k$ and a surjective $p$-rational map $f: \underline{H} \rightarrow \underline{H}^{*}$ whose kernel is $\underline{\operatorname{Ker}}(g)$. We get an induced $p$-rational map $\underline{g}^{*}: \underline{H}^{*} \rightarrow \underline{G}$ with $\underline{g}=\underline{g}^{*} f$. Further $f$ carries $\underline{H}(k)$ to $\underline{H}^{*}(k)$. Thus we may assume $\operatorname{Ker}(\underline{g})$ is trivial.

The image $\underline{g}(\underline{H})$ contains $A$, which is Zariski dense in $\underline{G}$, so it equals $\underline{G}$. Let $\underline{h}$ be the inverse map to $\underline{g}$. Then $\underline{h}$ is $p$-rational. Composing with a power of Frobenius (and changing $\underline{H}$ appropriately), we may assume $\underline{h}$ is rational.

Lemma 5.7. Let $\underline{G}$ be a semi-Abelian variety defined over $K, G=G(K)$. Let $A$ be a semi-pluriminimal definable subgroup of $G$. Then $A$ is (strongly) rigid.

Proof. By 4.6, 4.13, we may assume $A$ is semi-minimal. Further we may assume $A$ is Zariski dense in $\underline{G}$. If $A$ is locally modular, we are done by 4.11 . Otherwise by 5.6 there exist an algebraic group $\underline{H}$ defined over $k$, isogenous to $\underline{G}$, and a surjective map $h: A \rightarrow \underline{H}(k)$ with finite kernel. $\underline{H}$ is also a semi-Abelian variety, so $\underline{H}(k)$ is (strongly) rigid. By 4.14, $A$ is (strongly) rigid.

Proof of 5.1. The proof is now a sequence of reductions, leading to 5.6.

We may assume $X$ has finite stabilizer; otherwise we may quotient out the connected component of the stabilizer. Let $A$ be the maximal semi-pluriminimal connected subgroup of $\Gamma$. Then 4.3 (ii) holds. By 5.7, 4.3 (i) holds also. By assumption $X \cap \Gamma$ is Zariski dense in $X$. Choose a definable $Y \subseteq X \cap \Gamma$, such that $Y$ is Zariski dense in $X$, and of least possible dimension and multiplicity. Since $X$ is irreducible, whenever $Y$ is written as a finite union $\bigcup_{i} Y_{i}$, one of the sets $Y_{i}$ must be Zariski dense in $X$. Hence $Y$ has multiplicity one. Moreover if $Y^{\prime}$ is a subset of $Y$ of the same dimension, then $Y^{\prime}$ is Zariski dense in $X$, since the complement cannot be.

Observe that if translation by an element $a$ stabilizes $Y$ in the sense that $\operatorname{dim}(Y \cap(Y+a))=\operatorname{dim}(Y)$, then the Zariski closure of $Y \cap(Y+a)$ must be $X$, so (as $X \cap(X+a)$ is Zariski closed and contains $Y \cap(Y+a))$ the element $a$ stabilizes $X$ as a set. Thus the dimension-theoretic stabilizer of $Y$ is contained in the set-stabilizer of $X$; so it is finite.

Thus 4.3 (iii) is also true of $Y$. So by 4.3, a subset $Y^{\prime}$ of $Y$ of the same dimension is contained in a single coset $c+A$ of $A$. In particular $(c+A) \cap X$ is Zariski dense in $X$. Replacing $X$ by $X-c$, and $\Gamma$ by $A$, we may assume $\Gamma=A$ is semi-pluriminimal.

Write $A$ as a sum of orthogonal subgroups $A_{i}$, with $A_{i}$ semi-minimal. Let $B$ be the sum of all nonlocally modular $A_{i}$, and $C$ the sum of the rest. By 5.4, if $A_{i}, A_{j}$ are nonlocally modular, then they are nonorthogonal to $k$, and hence to each other, 
so $i=j$. Thus $B$ is semi-minimal. By 4.15, $Y$ is contained in a single coset of $B$. Translating, we may assume $Y$ is contained in $B$.

Let $\underline{G}$ be the Zariski closure of $B$. Then $\underline{G}$ is a group subvariety of $\underline{S}$, containing $X$ (the Zariski closure of $Y \subseteq B$ ). By 5.6, there exist an algebraic group $\underline{S}_{0}$ defined over $k$ and a bijective rational homomorphism $\underline{h}: \underline{G} \rightarrow \underline{S}_{0}$ defined over $K$; and $\underline{h}(B)=\underline{S}_{0}(k)$. So $S_{0}$ is semi-Abelian. Let $X_{0}$ be the Zariski closure of $\underline{h}(Y)$. Since $\underline{h}(Y) \subseteq \underline{h}(B) \subseteq \underline{S}_{0}(k), X_{0}$ is defined over $k$. Clearly $\underline{h}^{-1}\left(X_{0}\right)$ contains $X$. Since $\underline{h}$ is bijective, $\underline{h}^{-1}\left(X_{0}\right)=X$.

Remark 5.8. We observe that Theorem 5.1 for saturated $K$ implies the same statement for arbitrary models $K$. The assumptions that $\Gamma$ is of finite dimension and that $\Gamma \cap X$ is Zariski dense in $X$ should both be understood in the universal domain however. There are two points to observe here.

(i) Suppose $S$ is defined over $L$. The domain and kernel of $h$, being algebraic subgroups of $S$, are defined over a separable extension of $L\left(=L^{s}\right)$. Hence up to a $p$-rational isomorphism, $S_{0}$ is defined over $L$. Let $L^{*}$ be a saturated elementary extension of $L$, and let $k^{*}=\bigcap_{n} L^{* p^{n}}$ (in characteristic $p>0$ ) or $k^{*}=$ constants of $L^{*}$ (in the differential case). In either case $k^{*}, L$ are linearly disjoint over $k$. (In $\operatorname{char} p>0$, it is because $L^{* p^{n}}$ is linearly disjoint from $L$ over $L^{p^{n}}$ for each $n$.) Theorem 5.1 states that $S_{0}$ is defined over $k^{*}$. It follows that a $p$-birational copy of $S_{0}$ exists over the algebraically closed field $k$. More precisely, there exist $S_{0}^{\prime}$ defined over $k$, a surjective map $h^{\prime}: \operatorname{dom}(h) \rightarrow S_{0}^{\prime}$ defined over $L$, and a $p$-birational map $h^{\prime \prime}: S_{0}^{\prime} \rightarrow S_{0}$ defined over $k^{*}$, such that $h=h^{\prime \prime} h^{\prime}$. It follows similarly that $h^{\prime \prime-1} X_{0}=h(X+t)$ is defined over $k^{*} \cap L=k$. (The $t$ in $S$ such that $h(X+t)$ is defined over $k^{*}$ may be chosen in $L$.)

(ii) Note that if $\Gamma=\bigcap_{n} \Gamma_{n}$, where $\Gamma_{n}$ is a definable group, then $\Gamma \cap X$ is Zariski dense in $X$ iff, for each $n, \Gamma_{n} \cap X$ is Zariski dense in $X$. This is because $\Gamma \cap X$ is Zariski dense in $X$ if and only if, for each $m$, there exist $a_{1}, \ldots, a_{m}$ in $\Gamma \cap X$ such that $\left(a_{1}, \ldots, a_{m}\right)$ is a generic point of $X^{m}$ in the sense of algebraically closed fields. (And the compactness theorem of model theory applies.) Similarly one can deal with the case where for each $n, C_{n} \cap X$ is Zariski dense in $X$, where $C_{n}$ is some coset of $\Gamma_{n}$. In this case in the saturated extension there will be a coset of $\bigcap_{n} \Gamma_{n}$ meeting $X$ in a Zariski dense set.

Thus we have proved the following restatement of Theorem 5.1.

Theorem 5.9. Let $K$ be either a separably closed field of characteristic $p>0$, with a finite $p$-basis fixed, or a differentially closed field of char 0 . Let $k=\bigcap_{n} K^{p^{n}}$ if $p>0$, and $k=\{x: D x=0\}$ if the characteristic is 0 . Let $S$ be a semiAbelian variety defined over $K$. Let $\Gamma_{n}(n=1,2, \ldots)$ be a descending sequence of definable subgroups of $S$, such that $\bigcap_{n} \Gamma_{n}$ has finite dimension. Assume that for each $n$, for some coset $C_{n}$ of $\Gamma_{n}, X \cap C_{n}$ is Zariski dense in $X$. Then there exist a semi-Abelian variety $S_{0}$ defined over $k$, a subvariety $X_{0}$ of $S_{0}$ defined over $k$, and a rational homomorphism $h$ from a group subvariety of $S$ into $S_{0}$, such that $X=h^{-1}\left(X_{0}\right)+c$ for some $c$.

\section{A question of Voloch's}

We prove here a refinement of Theorem 1.1, conjectured by Voloch (Theorem 6.4). In characteristic 0, a somewhat weaker version was proved in [BV93]. It is related to a conjecture of Lang concerning integral points on an open affine 
subset of an Abelian variety (see [BV93] and [La91]). We also observe some uniformities that arise from our method of proof. In this section we use only basic model theory, really only the compactness theorem, in the style of A. Robinson.

For simplicity, as in 1.2, we will assume away the homomorphisms that occur in the conclusion of 1.1. Thus throughout this section, let $k$ be an algebraically closed field, $K$ an extension field, $\underline{A}$ an Abelian variety defined over $K$ with no nonzero homomorphic images defined over $k, \underline{X}$ a subvariety of $\underline{A}$, and $\Gamma$ a $p^{\prime}$ finitely generated subgroup of $A$. (There is no difficulty in working with semiAbelian varieties and special subvarieties as in 1.1; we restrict to the Abelian case for simplicity only.)

Recall:

Corollary 1.2. $\underline{X}(K) \cap \Gamma=\underline{Y}(K) \cap \Gamma$, where $\underline{Y}$ is a (reducible) subvariety of $\underline{X}$, equal to a finite union of translates of Abelian subvarieties of $\underline{A}$.

We wish to exploit the method of proof to observe a uniformity in the finite number involved.

Theorem 6.1. Let $k, K, \underline{A}, \underline{X}$ be as above. There exists a finite number of Abelian subvarieties $\underline{B}_{1}, \ldots, \underline{B}_{m}$ with the following property. For any extension field $K^{\prime}$ of $K$ and any $p^{\prime}$-finitely-generated subgroup $\Gamma$ of $\underline{A}\left(K^{\prime}\right)$ of rank $g$, there exists a union $\underline{Y}$ of a finite number $l$ of cosets of the $\underline{B}_{i}$, such that $\underline{X}\left(K^{\prime}\right) \cap \Gamma=\underline{Y}\left(K^{\prime}\right) \cap \Gamma$.

Moreover, $l$ depends only on $\underline{A}, \underline{X}$, and $g$ but not on the actual choice of $\Gamma$. In characteristic $p$, we have: $l \leq m p^{r g}$ for a certain fixed $r$ depending on $\underline{A}, \underline{X}$ alone.

Proof. We give the proof in characteristic $p$, the characteristic 0 case being analogous, using a derivation on $K$ over $k$. In 6.1 we may first replace $K$ by a finitely generated field, and then by the separable closure of that field. Then $k=\bigcap_{n} K^{p^{n}}$. We now have the following lemma:

Lemma 6.2. Let $K$ be a separably closed field, and let $\underline{A}$ be an Abelian variety defined over $K$, with no nonzero homomorphic images defined over $k=\bigcap_{n} K^{p^{n}}$. Then there exist integers $r, m$, and Abelian subvarieties $\underline{B}_{i}$ of $\underline{A}$ (at most $m$ of them) with the following property: For any coset $C$ of $p^{r} A(K)$, there exists a subvariety $\underline{Y}$ of $\underline{X}$ with $C \cap \underline{X}(K)=C \cap \underline{Y}(K)$; and $\underline{Y}$ is the union of at most $m$ cosets of some of the $\underline{B}_{i}$.

Proof. Note that for a given choice of $r, m$ and $\underline{B} *=\left\{\underline{B}_{i}\right\}_{i=1, \ldots, m}$, there exists a first order formula $\psi(y)=\psi(r, m, \underline{B} *)(y)$ such that: $\psi(c)$ holds in $K$ iff for every union $\underline{Y}$ of at most $m$ cosets of some of the $\underline{B}_{i},\left(c+p^{r} A(K)\right) \cap \underline{X}(K) \neq\left(c+p^{r} A(K)\right) \cap \underline{Y}(K)$. (The formula quantifies universally over the possible cosets of the $\underline{B}_{i}$.)

Note that as $r$ and $m$ grow bigger, the formula $\psi(c)$ grows stronger. Suppose for contradiction that there are no $r, m$ and Abelian subvarieties $\underline{B}_{i}$ as asserted in the theorem. For any choice of $r, m, \underline{B} *$ there exists $c$ with $\psi(r, m, \underline{B} *)(y)$. Hence by compactness, there exists an element $c$ in some elementary extension $K^{*}$ of $K$ such that all the formulas $\psi(r, m, \underline{B} *)$ hold of $c$. $K^{*}$ may be chosen countably saturated. Let $C=c+p^{\infty} A$. Then by Corollary 1.2, $C \cap \underline{X}\left(K^{*}\right)=C \cap \underline{Y}\left(K^{*}\right)$ for some finite union $\underline{Y}$ of cosets of $m$ Abelian subvarieties $\underline{B}_{i}$ of $\underline{A}$. In particular $C \cap \underline{X}\left(K^{*}\right) \subseteq \underline{Y}\left(K^{*}\right)$. By compactness, for some $r,\left(c+p^{r} A\right) \cap \underline{X}\left(K^{*}\right) \subseteq \underline{Y}$. So $\left(c+p^{r} A\right) \cap \underline{X}\left(K^{*}\right)=\left(c+p^{r} A\right) \cap \underline{Y}\left(K^{*}\right)$. Thus $\neg \psi(c)$ holds with $\psi=\psi\left(r, m,\left\{\underline{B}_{i}\right\}_{i}\right)$, a contradiction. 
We now finish the proof of 6.1 . We simply observe that as $\Gamma$ is $p^{\prime}$-generated by $g$ elements, so is $\left(\Gamma+p^{r} A(K)\right) / p^{r} A(K)$, hence it has size at most $p^{r g}$. So $\Gamma$ is contained in $p^{r g}$ cosets of $p^{r} A(K)$, and the theorem follows.

Remark 6.3. Assume the situation of 6.1 , but let $\underline{A}, \underline{X}$ vary within an algebraic family of Abelian varieties and subvarieties. Then the same proof shows that $k, m$ do not depend on the actual choice of $\underline{A}, \underline{X}$, and that the $\underline{B}_{i}$ also vary within an algebraic family.

We now assume given a discrete valuation $v$ of $K / k$. (In characteristic 0 the assumption of discreteness of $v$ is not needed; it can probably be eliminated in characteristic $p$ too.) Then one can define a $v$-adic distance $d_{v}(a, \underline{X})$ between a point $a$ of $A$ and a subvariety $\underline{X}$ of $\underline{A}$. We will define below a quantity $\lambda_{\underline{X}}(a)$, which should be considered as $-\log d_{v}(a, \underline{X})$. Thus 6.1 states that for $a \in \Gamma$, if $d_{v}(a, \underline{X})=0$ then also $d_{v}(a, \underline{Y})=0$, or equivalently that if $\lambda_{\underline{X}}(a)$ is infinite then $\lambda_{\underline{Y}}(a)$ is infinite. We prove a continuous version of this, conjectured by Voloch.

Theorem 6.4. With the assumptions above, there exist a finite union $\underline{Y}$ of translates of Abelian subvarieties of $\underline{A}$, and a constant $c$, such that: for all $a \in \Gamma$, $\lambda_{\underline{X}}(a) \leq c \cdot \lambda_{\underline{Y}}(a)$.

Remark 6.5. This statement naturally carries over to points of the $v$-adic closure of $\Gamma$ in $\underline{A}\left(K_{v}\right)$, where $K_{v}$ is the $v$-adic completion of $K$.

Definition. To define $\lambda$, we consider $\underline{A}$ as embedded in projective space $\mathbf{P}^{m}$. Let $R=\{t \in K: v(t) \geq 0\}$ be the valuation ring of $v$. Any point $x$ of $\mathbf{P}^{m}(K)$ can be written in projective coordinates as $x=\left(x_{0}: \cdots: x_{m}\right)$, with $v\left(x_{i}\right) \geq 0$ and $v\left(x_{i}\right)=0$ for some $i$. We let

$$
\begin{array}{r}
\lambda_{\underline{X}}^{\prime}(x)=\inf \left\{v\left(f\left(x_{0}: \cdots: x_{m}\right)\right): f\right. \text { a homogeneous polynomial in } \\
R[X] \text { vanishing on } X\} .
\end{array}
$$

Note that if $\left\{f_{j}\right\}$ are homogeneous polynomials generating the ideal of $\underline{X}$ in $R[X]$, then $\lambda_{\underline{X}}^{\prime}(x)=\min \left\{v\left(f_{j}\left(x_{0}: \cdots: x_{m}\right)\right): j\right\}$. We are interested in small distances from $\underline{X}$, hence large values of $\lambda^{\prime}$, so for convenience we let $\lambda_{\underline{X}}(x)=\max \left(1, \lambda_{\underline{X}}^{\prime}(x)\right)$.

Lemma 6.6. Let $K$ have characteristic $p>0, \underline{A}$ a group variety over $K$. Let $\underline{X}, \underline{Y}$ be subvarieties of $\underline{A}$ defined over $K$. Suppose $\underline{X}\left(K^{s}\right) \cap p^{r} A\left(\underline{K}^{s}\right)=\underline{Y}\left(K^{s}\right) \cap p^{r} \underline{A}\left(K^{s}\right)$. Then for some integer $c$ and for all $a \in p^{r} \underline{A}(K), \lambda_{\underline{X}}(a) \leq c \cdot \lambda_{\underline{Y}}(a)$.

Proof. Every separable extension of $K$ embeds over $K$ into some elementary extension of $K^{s}$. Hence the hypothesis implies that $\underline{X}\left(K^{\prime}\right) \cap p^{r} \underline{A}\left(K^{\prime}\right)=\underline{Y}\left(K^{\prime}\right) \cap p^{r} \underline{A}\left(K^{\prime}\right)$ for all separable extensions $K^{\prime}$ of $K$.

Suppose for contradiction that there is no integer $c$ as required. Consider the language describing an extension field $K^{*}$ of $K$, a valuation $v^{*}$ of $K^{*}$ extending $v$ on $K$, with value group $Z^{*}$ (extending the value group $\mathbf{Z}$ of $v$ ), and an element $a^{*}$ of $\underline{A}\left(K^{*}\right)$. We define $\lambda^{*}$ from $v^{*}$ as $\lambda$ is defined from $v$. The following statements are first-order:

(i) $a^{*}$ is in $p^{r} \underline{A}\left(K^{*}\right)$.

(ii) $v^{*}$ extends $v\left(v^{*}(\alpha)=v(\alpha)\right.$ for $\left.\alpha \in K\right)$. $Z^{*}$ is an ordered group with a least positive element (hence containing the integers $\mathbf{Z}$ as a convex subgroup).

(iii) $\lambda_{\underline{X}}^{*}\left(a^{*}\right)>c \lambda_{\underline{Y}}^{*}\left(a^{*}\right)(c=1,2, \ldots)$. 
By assumption any finite number of the above axioms can be satisfied (with $K^{*}=$ $K, v^{*}=v$ ). Hence by compactness there exist $K^{*}, v^{*}, a^{*}$ with these properties. Let $R^{*}$ be the valuation ring of $v^{*}$, and let

$$
M^{\prime}=\left\{x \in R^{*}: v^{*}(x)>n \lambda_{\underline{Y}}^{*}\left(a^{*}\right), \text { all } n\right\} .
$$

This is a prime ideal of $R^{*} \cdot v^{*}(x)=v(x) \in \mathbf{Z}$ for $x$ in $R^{*} \cap K$, so $M^{\prime}$ meets $R^{*} \cap K$ trivially; thus $K$ embeds into $K^{\prime}=R^{*} / M^{\prime}$.

$K^{\prime}$ is a separable extension of $K$. For suppose it is not. Let $t \in K, v(t)=1$. Then (since $K / k$ has transcendence degree 1 ) there is a $p$-th root of $t$ in $K^{\prime}$; i.e. there is $s$ in $R^{*}$ with $s^{p}-t$ in $M^{\prime}$, i.e. $v^{*}\left(s^{p}-t\right)$ large. But either $v^{*}(s)=0$ and $v^{*}\left(s^{p}-t\right)=0$, or $v^{*}(s) \geq 1$ so $v^{*}\left(s^{p}\right) \geq p$ and $v^{*}\left(s^{p}-t\right)=1$; a contradiction.

Let $a^{\prime}$ be the image of $a^{*}$ in $K^{\prime}$. By (iii) we have that $\lambda_{\underline{X}}\left(a^{*}\right)$ is in $M^{\prime}$, so $a^{\prime}$ is in $X\left(K^{\prime}\right)$. Since $a^{*}=p^{r} b^{*}$ for some $b^{*}$, and $b^{*}$ may be written with projective coordinates from $R^{*}$, we see that $a^{\prime}=p^{r} b^{\prime}$ for some $b^{\prime}$ in $\underline{A}\left(K^{\prime}\right)$. Thus by the first paragraph, $a^{\prime}$ is in $\underline{Y}\left(K^{\prime}\right)$. But $\lambda_{\underline{Y}}\left(a^{*}\right)$ is not in $M^{\prime}$, so $a^{\prime}$ is not in $\underline{Y}\left(K^{\prime}\right)$. This contradiction proves the lemma.

Lemma 6.7. Let there be given a derivation $D$ of $K$ over $k$ compatible with the valuation $v$, in the sense that for some constant $b$ in the value field, if $v(x)>b$, then $v(D x)>v(x)-b$. (We can take $b=1$.) Let $\underline{A}$ be a group variety over $K$, $\underline{X}, \underline{Y}$ be subvarieties of $\underline{A}$ defined over $K$, and $B$ be a subgroup of $\underline{A}(K)$ defined by a differential equation. Suppose $\underline{X}\left(K^{d}\right) \cap B=\underline{Y}\left(K^{d}\right) \cap B$ holds in some differential closure $K^{d}$ of $K$. Then for some integer $c$ and for all $a \in B(K), \lambda_{\underline{X}}(a) \leq c \cdot \lambda_{\underline{Y}}(a)$.

Proof. Entirely analogous to 6.6 (and indeed we could have used 6.7 to prove 6.6). We need only note that in an elementary extension $\left(K^{*}, D^{*}, v^{*}\right)$ of $(K, D, v)$, the derivation $D^{*}$ continues to satisfy that $v^{*}(x)>b$ implies $v^{*}\left(D^{*} x\right)>v^{*}(x)-b$, hence is continuous, and hence induces a derivation of $R^{*} / M^{\prime}$ of the proof of 6.6.

Proof of 6.4. Again we limit ourselves to giving the proof in characteristic $p>0$. We have $\Gamma \subseteq \Xi+p^{r} A(K)$ for some finite set $\Xi$. By 6.2 , for some finite union $\underline{Y}$ of translates of Abelian subvarieties of $\underline{A}$,

$$
\underline{X} \cap\left(\Xi+p^{r} A\left(K^{s}\right)\right)=\underline{Y} \cap\left(\Xi+p^{r} A\left(K^{s}\right)\right) .
$$

Thus by 6.6, for some $c$ and all $a \in\left(\Xi+p^{r} A\left(K^{s}\right)\right), \lambda_{\underline{X}}(a) \leq c \cdot \lambda_{\underline{Y}}(a)$. (Actually we require here a version of 6.4 valid for $\left(\Xi+p^{r} A\left(K^{s}\right)\right)$ in place of $p^{r} A\left(K^{s}\right)$; this can be proved in the same way.)

\section{REFERENCES}

[AV91] D. Abramovich and J. F. Voloch Toward a proof of the Mordell-Lang conjecture in characteristic $p$, Internat. Math. Res. Notices 5 (1992), 103-115. MR 94f: 11051

[Bu92] A. Buium, Intersections in jet spaces and a conjecture of S. Lang, Ann. of Math. (2) 136 (1992), 557-567. MR 93j: 14055

[Bu93] _ Effective bound for the geometric Lang conjecture, Duke Math. J. 71 (1993), 475-499. MR 95c:14055

[BV93] A. Buium and J. F. Voloch, Integral points of abelian varieties over function fields of characteristic zero, Math. Ann. 297 (1993), 303-307. MR 94i:14029

[Del88] F. Delon, Idéaux et types sur les corps séparablement clos, Supplément au Mém. Soc. Math. France (N.S.), vol. 116, no. 33, Soc. Math. France, Paris, 1988. MR 90m:03067

[Hr90] E. Hrushovski, Unidimensional theories are superstable, Ann. Pure Appl. Logic 50 (1990), 117-138. MR 92g:03052

[HP86] E. Hrushovski and A. Pillay, Weakly normal groups, Logic Colloquium '85, NorthHolland, Amsterdam, 1987. MR 88e:03051 
[HS] E. Hrushovski and Z. Sokolovic, Minimal subsets of differentially closed fields, Trans. Amer. Math. Soc. (to appear).

[HZ] E. Hrushovski and B. Zil'ber, Zariski geometries, J. Amer. Math. Soc. 9 (1996), 1-56. CMP 95:06

[HZ93] , Zariski geometries, Bull. Amer. Math. Soc. (N.S.) 28 (1993), 315-323. MR 93j:14003

[La65] S. Lang, Division points on curves, Ann. Mat. Pura Appl. (4) 70 (1965), 229-234. MR 32:7560

[La91] _ Number Theory III: Diophantine geometry, Encyclopaedia Math. Sci., vol. 60, Springer-Verlag, Berlin, Heidelberg, and New York, 1991. MR 93a:11048

[Las] D. Lascar, Rank and definability in superstable theories, Israel J. Math. 23 (1976), 53-87. MR 53:12931

[Ma58] Yu. Manin, Algebraic curves over fields with differentiation, Izv. Akad. Nauk SSSR Ser. Mat. 22 (1958), 737-756; English transl., Amer. Math. Soc. Transl. Ser. 2, vol. 37, Amer. Math. Soc., Providence, RI, 1964, pp. 59-78. MR 21:2652

[Ma63] , Rational points of algebraic curves over function fields, Izv. Akad. Nauk SSSR Ser. Mat. 27 (1963), 1395-1440; English transl., Amer. Math. Soc. Transl. Ser. 2, vol. 59, Amer. Math. Soc., Providence, RI, 1966, pp. 189-234. MR 28:1199

[Mes] M. Messmer, Groups and fields interpretable in separably closed fields, preprint.

[NeP89] A. Pillay, Model theory, stability theory, and stable groups, The Model Theory of Groups (A. Nesin and A. Pillay, eds.), Notre Dame Math. Lectures, no. 11, Univ. Notre Dame Press, Notre Dame, IN, 1989, pp. 1-22.CMP 21:09

[RR75] A. Robinson and P. Roquette, On the finiteness theorem of Siegel and Mahler concerning diophantine equations, J. Number Theory 7 (1975), 121-176. MR 51:10222

[Sa72] G. Sacks, Saturated model theory, W. A. Benjamin, Reading, MA, 1972. MR 53:2668

[So92] Z. Sokolovic, Model theory of differential fields, Ph.D. Thesis, Notre Dame, July, 1992.

[Weil48] A. Weil, Variétés abéliennes et courbes algébriques, Hermann, Paris, 1948. MR 10:621d

[Wood79] C. Wood, Notes on the stability of separably closed fields, J. Symbolic Logic 44 (1979), 412-416. MR 81m:03042

ABstract. We give a proof of the geometric Mordell-Lang conjecture, in any characteristic. Our method involves a model-theoretic analysis of the kernel of Manin's homomorphism and of a certain analog in characteristic $p$.

Department of Mathematics, Massachusetts Institute of Technology, 2-277, CamBRIDGe, MASSACHUSETTS 02139

Current address: Department of Mathematics, Hebrew University, Jerusalem, Israel

E-mail address: ehud@math.mit.edu 